\title{
A preliminary study of medial temporal lobe function in youths with a history of caregiver deprivation and emotional neglect
}

\author{
Françoise S. Maheu \\ Research Centre of the CHU Ste-Justine, Montreal, Quebec, Canada \\ and University of Montreal, Montreal, Quebec, Canada \\ MARY DOZIER \\ University of Delaware, Newark, Delaware \\ Amanda E. GuYer ANd DarCy Mandell \\ National Institute of Mental Health, Bethesda, Maryland \\ Elizabeth Peloso \\ University of Delaware, Newark, Delaware \\ Kaitlin Poeth and Jessica Jenness \\ National Institute of Mental Health, Bethesda, Maryland \\ JENNIFER Y. F. LAU \\ University of Oxford, Oxford, England \\ JoHN P. ACKerMan \\ University of Maryland School of Medicine, Baltimore, Maryland \\ AND \\ Daniel S. Pine and MoniQue Ernst \\ National Institute of Mental Health, Bethesda, Maryland
}

\begin{abstract}
Previous research findings have linked caregiver deprivation and emotional neglect with sensitivity to threatening cues. The present preliminary study investigated whether dysfunctions of the medial temporal lobe could underlie these associations. Using fMRI, we measured medial temporal lobe responses to emotional faces (angry, fearful, happy, neutral) among 30 youths. Eleven of the youths had a history of caregiver deprivation and emotional neglect. Attention states (i.e., attention to anger, fear, or physical attributes, or passive viewing) were systematically manipulated. Relative to comparison youths, youths with a history of caregiver deprivation and emotional neglect showed significantly greater left amygdala and left anterior hippocampus activation during the processing of threatening information. To our knowledge, these findings are the first to demonstrate altered medial temporal lobe function during the processing of threat cues in youths with a history of caregiver deprivation and emotional neglect.
\end{abstract}

Adverse experiences early in life are known to produce impairments in the emotional development of youths (Gunnar, Bruce, \& Grotevant, 2000; Teicher, Andersen, Polcari, Anderson, \& Navalta, 2002). In humans, research on early adverse experiences has typically focused on neglected and abused youths (see, e.g., Cicchetti \& Toth, 2005; Pollak, 2005; Teicher et al., 2002), as well as on youths with a history of caregiver deprivation - for example, youths who were separated from their primary caregivers and placed in foster care or orphanages (e.g., Dozier et al., 2006; Rutter \& the English and Romanian Adoptees Study Team, 1998; Rutter, O'Connor, \& the English and Romanian Adoptees Study Team, 2004). It is not surprising that caregiver deprivation is associated with emotional problematic outcomes since the attachment between caregivers and infants, which develops during the first year of life, plays a critical role in youths' survival and healthy emotional adaptation (see Dozier, Albus, Fisher, 
\& Sepulveda, 2002; Gunnar et al., 2000). Indeed, from an evolutionary perspective, there may be nothing more threatening for a young child than the lack or loss of a trusted primary caregiver (see Dozier et al., 2002; Gunnar et al., 2000). Additionally, experiences of caregiver deprivation are often associated with poor conditions of care as well as with abuse or neglect, either from primary caregivers or during institutional (orphanage) placement (see Dozier et al., 2002; Gunnar et al., 2000). Studies focusing on the influence of early maltreatment have reported that neglect (emotional, physical, or medical) and abuse (emotional, physical, or sexual) were also related to affective difficulties and adverse emotional adaptation (De Bellis, 2005; Gunnar et al., 2000; Teicher et al., 2002).

Caregiver deprivation, as well as neglect and abuse, are thought to impair emotional development by altering youths' abilities to process efficiently emotional information - particularly threatening cues - and by increasing risk for psychopathology. As such, postinstitutionalized youths, as well as youths in foster care, show difficulties in recognizing different facial emotional expressions, especially fear and anger (Masten et al., 2008; Pears \& Fisher, 2005b; Vorria et al., 2006; Wismer-Fries $\&$ Pollak, 2004). Similar problems were observed in neglected youths, whereas abused youths showed enhanced sensitivity to threatening stimuli (Pine et al., 2005; Pollak, 2003; Pollak, Cicchetti, Hornung, \& Reed, 2000; Pollak \& Sinha, 2002; Pollak \& Tolley-Schell, 2003). In addition, studies in postinstitutionalized and foster care youths, as well as in neglected and abused youths, reported that levels of anxiety and major depression disorders are particularly elevated in these populations as compared with those in comparison youths (American Academy of Pediatrics, 2000; De Bellis, 2005; Kaufman, Plotsky, Nemeroff, \& Charney, 2000; Pine, 2003; Sawyer, Carbone, Searle, \& Robinson, 2007; Tyler, Allison, \& Winsler, 2006). Such mental health conditions carry long-term consequences, since they are highly predictive of adult suicidal ideation, anxiety, and major depression disorders (Pine, 2003, 2007; Pine, Cohen, Gurley, Brook, \& Ma, 1998).

In recent years, it has been suggested that the medial temporal lobe (encompassing the amygdala and hippocampus) may be particularly sensitive to early experiences of caregiver deprivation, neglect, and abuse in animals and humans (e.g., Bremner, 2007; De Bellis, 2005; De Bellis \& Thomas, 2003; McGowan et al., 2009; Mehta et al., 2009; Rutter et al., 2007; Sanchez, Ladd, \& Plotsky, 2001; Teicher et al., 2002). Indeed, the medial temporal lobe plays a crucial role in mediating emotional processes such as sensitivity to threatening cues as well as anxiety and mood disorders (Adolphs, 2003; Davidson, 2004).

In the present preliminary study, we examined medial temporal lobe function during emotion processing both in youths who experienced caregiver deprivation before their adoption by U.S. families and in comparison youths. All youths with a history of caregiver deprivation had experienced emotional neglect before being adopted.

To date, only three studies have investigated the influence of caregiver deprivation and foster care/institutionalization on brain structure and function in youths. Findings from these studies provide support for perturbations within the medial temporal lobe. Recently, Mehta et al. (2009) reported preliminary structural neuroimaging findings in a sample of 14 caregiver-deprived youths who were institutionalized in Romania. As compared with 11 noninstitutionalized controls, the caregiver-deprived youths showed greater amygdala volume, but no differences were observed in hippocampal volume. In the second study, Eluvathingal et al. (2006) used diffusion tensor imaging and reported reduced integrity of the uncinate fasiculus (white matter tracts connecting the inferior frontal lobe to the anterior temporal lobe [including the amygdala]), in 7 caregiverdeprived youths who were adopted from Eastern European orphanages as compared with 7 comparison youths. Finally, reduced glucose metabolism using PET during a resting state was observed in the amygdala and hippocampus of 10 postinstitutionalized Romanian children as compared with that in healthy adults and comparison children with medically intractable epilepsy (Chugani et al., 2001).

In contrast with the scarce amount of work performed in postinstitutionalized youths, a greater number of neuroimaging studies have been conducted with neglected and abused youths and adults. Experiences of foster care or institutionalization were not reported for the youths and adults who were investigated in these studies. However, anxiety disorders were observed in all of the participants of these studies. Structural neuroimaging findings have reported smaller right temporal lobe volume (De Bellis et al., 2002) and altered hippocampal volume (decreased volume, Carrion, Weems, \& Reiss, 2007; increased volume, Tupler \& De Bellis, 2006) in anxious pediatric patients with a history of neglect and abuse. Medial temporal lobe function was not measured in these pediatric patients. In adults, patients with anxiety disorders related to childhood neglect and abuse have been found to have abnormally low hippocampal activation and abnormally high amygdala activation in response to threat cues (Bremner, 2007; Bremner et al., 1999; Bremner et al., 2005; Bremner, Vythilingam, Vermetten, Southwick, McGlashan, Nazeer, et al., 2003; Bremner, Vythilingam, Vermetten, Southwick, McGlashan, Staib, et al., 2003).

In the present study, we aimed at collecting preliminary data on medial temporal lobe function in a sample of youths who had experienced caregiver deprivation and emotional neglect before being adopted. Given the difficulty of recruiting such youths, the goal of this preliminary study was to examine the functioning of the different nodes of the medial temporal lobe (amygdala, hippocampus) during emotion processing in order to generate hypotheses for future research with this population. Understanding these links while individuals are still young may help to develop early interventions that would effectively normalize a disrupted development trajectory early in its course, before emotional difficulties become chronic.

To probe medial temporal lobe activation, we used an fMRI emotional face-viewing paradigm presenting four different types of emotional faces (fearful, angry, happy, neutral faces) under four different types of attentional states (How afraid of the face are you?; How hostile is the face?; How wide is the nose of the face?; passive viewing). 
Hence, this task manipulates attention toward (How afraid are you?) and away from subjective feelings of fear (all of the three other attention states). This specific task was chosen because it has been shown to reliably engage the medial temporal lobe in healthy youths and adults (Guyer et al., 2008; McClure et al., 2004; Monk et al., 2003; Nelson et al., 2003), as well as in different pediatric patient populations with disorders, including anxiety (McClure et al., 2007; Pérez-Edgar et al., 2007; Roberson-Nay et al., 2006), depression (Roberson-Nay et al., 2006), and steroid dysfunction (e.g., Ernst et al., 2007; Maheu et al., 2008). Given that caregiver-deprived as well as neglected and abused youths are at risk for anxiety and major depression disorders, this task was expected to engage medial temporal lobe function effectively in the population of the present study.

Using this fMRI emotional face-viewing paradigm and, on the basis of the aforementioned previous research, we hypothesized that, as compared with youths who were reared by their biological parents (comparison youths), caregiver-deprived and emotionally neglected youths would differ in the activation of the medial temporal lobe during the processing of threat cues - that is, fearful and angry faces versus neutral faces when attention was focused on subjective feelings of fear.

Specifically, we expected that medial temporal lobe activation would differ between groups when participants attended to subjective feelings of fear, because two recent studies using this paradigm found that youths with, or at high risk for, anxiety disorders had exaggerated amygdala response to threatening faces only when attention was focused on internal feelings of fear (see McClure et al., 2007; Pérez-Edgar et al., 2007). These findings suggest that a subjective fear attention state may be necessary to activate structures of the medial temporal lobe during the processing of threat cues in youths with emotional difficulties similar to those observed in caregiver-deprived and emotionally neglected youths. However, not enough is currently known about the effect of early caregiver deprivation or emotional neglect on medial temporal lobe function to predict the direction of the hypothesized perturbations (increased or decreased activation).

\section{METHOD}

\section{Participants}

A total of 30 youths completed the study. Eleven ( 3 boys; age range $=9-18$ years) youths had a history of caregiver deprivation and emotional neglect, whereas 19 ( 5 boys; age range $=9-18$ years) youths had not experienced caregiver deprivation or any form of neglect or abuse. Demographic characteristics for the youths with a history of caregiver deprivation and emotional neglect as well as for the comparison youths are presented in Table 1. Youths with a history of caregiver deprivation and emotional neglect experienced life in either U.S. foster care $(n=4)$ or international orphanages ( $n=7$; in Russia, Serbia, China, or Korea) before their adoption in U.S. families. None of the caregiver-deprived and emotionally neglected youths had received any past or present psychotherapeutic or pharmacologic treatment. For the present study, which was performed in collaboration with the Infant Caregiver Project at the University of Delaware (Dozier et al., 2006), youths were recruited from newspaper announcements and from announcements to foster care and adoptive care agencies. All of the fMRI data were collected at the National Institute of Mental Health (NIMH), and the study was approved by the institutional review boards of the NIMH and University of Delaware. Prior to participation in the study, parents and youths gave written consent and assent, respectively. All participants were compensated for their participation in the study, following the guidelines provided by the NIMH.

Information on caregiver deprivation and emotional neglect was gathered using a modified version of the Schedule for Affective Disorders and Schizophrenia for School-Age Children-Present and Lifetime Version (K-SADS-PL; Kaufman et al., 1997) with the adoptive parents. Questions concerning traumatic events over the participant's lifetime were incorporated into an expanded version of the trauma section included in the K-SADS-PL. These additional questions involving the types of interpersonal and noninterpersonal traumas and the nature and circumstances of such traumatic experiences are described in the K-SADS-PL. Adoptive parents commented on their child's history of caregiver deprivation and emotional neglect by reporting information obtained from well-documented records that were shared with them by caseworkers. Examples of emotional

Table 1

Demographic and Clinical Characteristics of Youths With a History of Caregiver Deprivation and Emotional Neglect and of Comparison Youths

\begin{tabular}{|c|c|c|c|c|}
\hline & \multicolumn{2}{|c|}{$\begin{array}{c}\text { Youths With } \\
\text { a History of } \\
\text { Caregiver } \\
\text { Deprivation and } \\
\text { Emotional Neglect } \\
\text { (3 boys, } 8 \text { girls) } \\
\end{array}$} & \multicolumn{2}{|c|}{$\begin{array}{c}\text { Comparison } \\
\text { Youths } \\
\text { ( } 5 \text { boys, } 14 \text { girls })\end{array}$} \\
\hline & $M$ & $S D$ & $M$ & $S D$ \\
\hline Age (years) & 13.75 & 2.32 & 13.41 & 2.70 \\
\hline Age range & \multicolumn{2}{|c|}{$9-18$} & \multicolumn{2}{|c|}{$9-18$} \\
\hline Tanner stage & 3.80 & $0.63^{\mathrm{a}}$ & 3.21 & 1.36 \\
\hline Wechsler IQ & 107.73 & 8.64 & 108.16 & 14.27 \\
\hline SES & 42.45 & 13.66 & 50.07 & 17.31 \\
\hline Clinical Depression Inventory score & 39.64 & 4.67 & 41.24 & $7.28^{\mathrm{b}}$ \\
\hline Screen for Child Anxiety Related & & & & \\
\hline Emotional Disorders questionnaire score & 17.00 & 8.69 & 14.13 & $9.14^{\mathrm{c}}$ \\
\hline
\end{tabular}

Note-Tanner puberty stages: On a scale of 1 to 5 , with $1=$ body is in the prepuberty stage and $5=$ puberty is reached, body is adult-like. SES, socioeconomic status, determined by the four-factor Hollingshead scale (Hollingshead, 1973); lower scores indicate higher SES. ${ }^{a} n=10$ because 1 participant refused to have her puberty stage evaluated. $\mathrm{b}_{n}=$ 17 because of data lost. ${ }^{c} n=15$ because of data lost. 
neglect included inadequate attention to the children's needs for attention and affection, young children left in room alone crying for an extended period of time, and biological mothers who had parties in the home during which people abused substances.

All caregiver-deprived and emotionally neglected youths ranged in the age of their first placement in foster care or international orphanage from less than 1 month to 72 months old (mean age at first placement $=19.54$ months, $S D=24.35$ months). Youths had been placed in U.S. foster care or international orphanages between one to three times before their final adoption, with a mean of $1.81(S D=$ 0.87 ) placements. Youths had resided in U.S. foster care or international orphanages for an average of $47.95(S D=31.67)$ months prior to their adoptive placement (range $=4-120$ months). The age at adoption ranged from 4 months to 132 months old (mean age at adoption $=71.68$ months, $S D=44.31$ months). Youths had been residing in their adoptive homes for an average of 8 years $(M=$ 98.96 months, $S D=41.17$ months; range $=46-170$ months) at the time of the present study.

All of the participants underwent a physical examination. Caregiverdeprived and emotionally neglected youths as well as comparison youths were all clear of chronic medical conditions and were not taking any medication. The Tanner puberty stage (Tanner \& Whitehouse, 1976), evaluated on a scale of $1=$ prepuberty stage to $5=$ fully mature, was determined by a pediatrician in caregiver-deprived and emotionally neglected youths, and by a self-administered questionnaire in the comparison group (Duke, Litt, \& Gross, 1980). Psychiatric status was assessed using the K-SADS-PL (Kaufman et al., 1997) with adoptive parents and youths, separately. Two youths with a history of caregiver deprivation and emotional neglect met criteria for at least one psychiatric diagnosis: lifetime and ongoing specific phobia $(n=1)$, and lifetime and ongoing separation anxiety disorder and social phobia $(n=1)$. Comparison youths were free of any past or present psychiatric disorders, and they had no history of neglect or abuse (as evaluated by the trauma section of the K-SADS-PL). The Clinical Depression Inventory (CDI; Kovacs, 1985; Smucker, Craighead, Craighead, \& Green, 1986) and the Screen for Child Anxiety Related Emotional Disorders questionnaire (SCARED; Birmaher et al., 1997) were administered to all of the participants to quantify the severity of the symptoms of anxiety or depression (Table 1). Exclusion criteria were current Tourette's syndrome, obsessivecompulsive disorder, suicidal ideations, lifetime history of mania or psychosis, pervasive developmental disorder, neurological disorders or head injury, and an IQ less than 70, as assessed by the Wechsler Abbreviated Scale of Intelligence for Children (Wechsler, 1999). Socioeconomic status (SES) was determined by the four-factor Hollingshead scale (Hollingshead, 1973) in both groups (lower scores on the Hollingshead scale indicate higher SES).

The groups did not differ on age $[t(28)=-0.35, p=.73]$, sex distribution $\left[\chi^{2}(1)=0.003, p=.95\right]$, Tanner stage $\left[\chi^{2}(4)=3.48\right.$, $p=.48]$, IQ $[t(28)=0.09, p=.93]$, SES $[t(28)=1.25, p=.22$; see Table 1], anxiety scores (SCARED) $[t(23)=0.77, p=.45]$, or depression scores $(\mathrm{CDI})[t(25)=0.52, p=.61]$.

\section{Face-Viewing Paradigm}

Stimuli were selected from three standardized sets of grayscale photographs depicting different facial expressions that were constructed by Ekman and Friesen (1976), Gur (www.uphs.upenn.edu/ bbl/pubs/downloads/nptasks.shtml), and Tottenham and Nelson (www .macbrain.org/faces/index.htm). The photographs comprised 32 portraits of adult actors who were selected randomly from a larger pool of 56 actors. Each actor presented one of four emotional expressions (angry, fearful, happy, or neutral) throughout the entire paradigm, and 8 different actors were viewed for each expression. Whereas facial expressions for a given actor were held constant within each participant's task, expressions varied randomly across different participants' tasks. Thus, 1 participant may have viewed a given actor displaying a happy expression consistently, whereas a subsequent participant may have viewed the same actor displaying an angry expression consistently. This feature controlled for variability in nonemotional aspects of the actors (e.g., ethnicity, hair texture). Female actors were used in half of the photographs to control for sex. While in the scanner, participants viewed the series of 32 adult faces ( 8 angry, 8 fearful, 8 happy, 8 neutral) under four attention conditions. Three attention conditions required participants to attend to different aspects of the face stimuli: (1) How afraid does the face make you feel? (2) How hostile does the face appear? (3) How large is the nose? These questions were rated on a five-key button box $(1=$ not very to $5=$ extremely $)$. In the fourth condition, participants' attention was unconstrained, so that the faces were viewed passively without participants making any ratings. For baseline comparison, 32 trials of fixation crosses were presented randomly within each attention condition.

The face-viewing paradigm followed a rapid event-related design and was presented as a 14.2-min single run comprising 160 trials ( 32 faces $\times 4$ conditions, plus 32 trials presenting a fixation point). Each facial expression was presented a total of four times, once during each of the four conditions. Trials within a given condition were blocked, and the presentation order of blocks and facial expressions within blocks was randomized across participants. Rating instructions appeared for $3 \mathrm{sec}$ before each condition block. Faces and fixation trials were shown for $4 \mathrm{sec}$ each and were followed by an intertrial interval showing a blank screen with a 750 - to 1,250 -msec jitter.

Stimuli were displayed using Avotec Silent Vision Glasses (Avotec, Inc., Stuart, FL), and responses were recorded via a five-key buttonbox (MRI Devices, Waukesha, WI). Participants were trained in an MRI simulator prior to entering the scanner to become familiar with the actual MRI environment and response device. Participants were also administered a practice version of the task to ensure understanding of the task. The practice version contained only photos of neutral facial expressions that were not shown in the MRI scanner.

\section{fMRI Data Acquisition}

Whole-brain BOLD fMRI data were acquired on a General Electric Signa 3-Tesla magnet (General Electric Medical Systems, Waukesha, WI). Head movement was constrained by the use of foam padding. Following sagittal localization and a manual shim procedure, functional $\mathrm{T} 2 *$-weighted images were gathered using an echoplanar single-shot gradient echo pulse sequence with a matrix size of $64 \times 64$, a repetition time (TR) of 2,000 msec, echo time (TE) of $40 \mathrm{msec}$, a field of view (FOV) of $240 \mathrm{~mm}$, and voxels of $3.75 \times$ $3.75 \times 5 \mathrm{~mm}$, providing whole brain coverage. Echo-planar images (EPI) were acquired in 23 contiguous $5-\mathrm{mm}$ axial slices per brain volume that were positioned parallel to the anterior commissure and posterior commissure (AC-PC) line. Following EPI data collection, a high-resolution T1-weighted anatomical image was acquired for each participant using a standardized magnetization-prepared gradient echo sequence (180 1-mm sagittal slices; FOV = 256; number of excitations $[\mathrm{NEX}]=1$; $\mathrm{TR}=11.4 \mathrm{msec}$;E $=4.4 \mathrm{msec}$; matrix size of $256 \times 256$; time to inversion [TI] $=300 \mathrm{msec}$; bandwidth $=$ $130 \mathrm{~Hz} /$ pixel; $33 \mathrm{kHz} / 256$ pixels) to facilitate spatial normalization.

\section{fMRI Data Processing}

Preprocessing and analyses were performed using the Statistical Parametric Mapping software (SPM99, Wellcome Department of Cognitive Neurology, London) and supplemental routines that were written in MATLAB 6 (The MathWorks, Inc., Natick, MA). Imaging data for participants who moved more than $3 \mathrm{~mm}$ in any plane, as assessed with MedX software (Medical Numerics, Sterling, VA), were excluded. Preprocessing procedures included corrections for slice timing and motion, coregistration to the anatomical data, and spatial normalization to a Montreal Neurologic Institute (MNI) T1weighted template image supplied with SPM99.

Event-related response amplitudes at the individual participant level for each event type in each attention set were estimated using a general linear model (GLM). The waveform used to model eventrelated responses was a rectangular pulse ( $4 \mathrm{sec}$ duration) that was convolved with the hemodynamic response function specified in SPM99. Contrast images were created for each participant using pairwise comparisons of the different event-related BOLD response 
amplitudes across conditions. Before performing group-level analyses, each contrast image was divided by the participant-specific voxel time series mean, generating values that were proportional to percentage fMRI signal change (Zarahn, Aguirre, \& D'Esposito, 1997). These normalized contrast images were then smoothed with an isotropic Gaussian kernel (FWHM $=8 \mathrm{~mm}$ ) to reduce nonstationarity in the spatial autocorrelation structure produced by the previous step (Friston, Mechelli, Turner, \& Price, 2000).

\section{Data Analysis}

Behavioral data. Behavioral measures (subjective ratings and RTs) to fearful, angry, happy, and neutral faces were collected during the hostile, afraid, and nose attention conditions (by design, behavioral data were not collected during passive viewing) and were submitted to group comparisons.

Two three-way repeated measures ANOVAs with emotional faces (angry, fearful, happy, neutral) and attention states (hostile, afraid, nose) as within-subjects factors and group (youths with a history of caregiver deprivation and emotional neglect vs. comparison youths) as the between-subjects factor were conducted on the dependant variables subjective ratings and RTs, respectively. When sphericity assumptions were not met, Greenhouse-Geisser (1959) corrections were applied to minimize the risk of Type 1 errors.

Imaging data. Because we were interested in comparing the reactivity of the medial temporal lobe between caregiver-deprived and emotionally neglected youths versus comparison youths, our a priori hypotheses motivated a region-of-interest (ROI) analysis based on the amygdala (left and right), anterior hippocampus (left and right), and posterior hippocampus (left and right). Both anterior and posterior hippocampi are implicated in emotion regulation; however, their processing of emotional information is functionally different. The anterior hippocampus is involved in the processing of anxiety- and threatrelated stimuli (Bannerman et al., 2004; Dolcos, LaBar, \& Cabeza, 2004), whereas the posterior hippocampus is involved in providing contextual memories to emotional stimuli (Bannerman et al., 2004; LeDoux, 2000). The ROIs were ascertained from standard anatomical criteria on a single MNI template and were applied to all normalized brains at the group level (Szeszko et al., 1999; Szeszko et al., 2002). Voxelwise tests were conducted in these anatomically defined volumes of interest. Consistent with the current standards (Hariri, Bookheimer, \& Mazziotta, 2000; Winston, Strange, O'Doherty, \& Dolan, 2002), we utilized the Gaussian random field threshold ( $\alpha=.05$, corrected) with small volume correction (SVC) implemented in SPM99. The statistical significance of activation in the ROIs was set to $p<.05$.

Group differences in task-related fMRI activation were computed on the a priori contrasts of interest that compared activation during fearful faces versus neutral faces, as well as during angry faces versus neutral faces, in the "how afraid" attention state (i.e., afraid-fearful faces vs. afraid-neutral faces and afraid-angry faces vs. afraid-neutral faces contrasts). Selection of these contrasts was based on two factors. First, prior research suggested that a subjective fear attention state may be necessary to activate structures of the medial temporal lobe in youths with emotional difficulties that are similar to those observed in caregiver-deprived and emotionally neglected youths (McClure et al., 2007; Pérez-Edgar et al., 2007). Second, the contrasts fearful faces versus neutral faces and angry faces versus neutral faces were chosen because fearful and angry faces have consistently been shown to engage medial temporal lobe activation in healthy and clinical populations (see, e.g., Armony, Corbo, Clément, \& Brunet, 2005; Dolan \& Fullam, 2009; Holt et al., 2005; B. T. Lee et al., 2008; McClure et al., 2004; Monk et al., 2003; Monk et al., 2008; Nelson et al., 2003; Reinders et al., 2006). In addition, amygdala and hippocampus activation have been observed selectively in response to fearful and angry faces in youths with anxiety disorders (McClure et al., 2007; Monk et al., 2008) — that is, in youths who show emotional difficulties related to caregiver deprivation and emotional neglect (Pine, 2003; Pine \& Cohen, 2002).

Data analyses proceeded in two stages. First, we examined grouplevel differences in the activation of the amygdala (left and right), anterior hippocampus (left and right), and posterior hippocampus (left and right) for the contrasts of interest-afraid-fearful faces versus afraid-neutral faces, and afraid-angry faces versus afraidneutral faces - using an ROI voxelwise SPM analysis. Second, through post hoc analyses that were conducted with SPSS-14.0, we examined the selectivity of any significant findings with respect to the different types of emotional faces and attention states. These secondary analyses examined the degree to which initial results were specific to fearful and angry faces and to the afraid attention state. To this end, the fourth emotion face-happy-and the other three attention states (How hostile is the face?; How wide is the nose?; passive viewing) were included in the analyses. These analyses were based on extracted individual signal change values at the peak voxel coordinates of the structures with significant SPM findings. SPM values were extracted for all 16 emotional faces $X$ attention states combination versus fixation (e.g., afraid-fearful faces vs. fixation, afraid-angry faces vs. fixation, afraid-happy faces vs. fixation, afraid-neutral faces vs. fixation, hostile-fearful faces vs. fixation, hostile-angry faces vs. fixation, etc.). These values were then analyzed using ANOVAs in SPSS. This method followed procedures established in previous work (McClure et al., 2007; PérezEdgar et al., 2007), allowing systematic analyses of the variables (emotional faces, attention states) that may have modulated the activation.

These analyses of the extracted peak voxel values included repeated measures ANOVAs with group (youths with a history of caregiver deprivation and emotional neglect vs. comparison youths) as the between-subjects factor, and emotional faces (angry, fearful, happy, neutral) and attention states (afraid, hostile, nose width, passive) as the within-subjects factors. Greenhouse-Geisser (1959) corrections were performed when appropriate.

Bivariate Pearson correlation analyses. Since the participants' age range (9-18 years) was relatively wide, we examined how age may have influenced peak voxels' activation in the medial temporal lobe. We also assessed how significant peak voxels' activation was modulated by characteristics of caregiver deprivation and adoption. These characteristics included the number of foster care or orphanage placements, the age at first placement in foster care or institution, the duration (in months) of foster care or institutionalization, the age when placed in the adoptive family, and the time (in months) spent in the adoptive family since being placed in the family. Correlation analyses performed with characteristics of caregiver deprivation or adoption-related variables were run in youths with a history of caregiver deprivation and emotional neglect only, because of the nature of these variables. All of the variables used in the correlation analyses met correlation assumptions.

\section{RESULTS}

\section{Performance Data}

Means and standard deviations of the subjective rating scores and RTs are presented by group in Table 2 . With respect to subjective ratings, there were significant main effects of emotional faces $[F(1.86,52.02)=37.35$, $p=.0001]$ and attention states $[F(2,56)=19.56, p=$ $.0001]$, which were subsumed by a significant two-way interaction of emotional faces $\times$ attention states $[F(3.78$, $105.87)=20.29, p=.001]$. As was expected, post hoc analyses revealed that angry faces received the highest "hostile" ratings and the lowest "nose width" ratings (all $p$ s $<.05)$. Happy faces received the highest "nose width" ratings but the lowest "hostile" and "afraid" ratings (all $p$ s $<.05$; see Table 2). Contrary to expectations, the ANOVA revealed no significant influence of group as a main effect or in interaction with other variables (all $F \mathrm{~s}<$ 0.92 , all $p \mathrm{~s}>.36$ ). 
Table 2

Means and Standard Deviations of Subjective Ratings and Reaction Times (RTs) for Each Emotional Face Type Under Each Attention State for Youths With a History of Caregiver Deprivation and Emotional Neglect, and for Comparison Youths

\begin{tabular}{|c|c|c|c|c|c|c|c|c|}
\hline & \multicolumn{4}{|c|}{ Subjective Rating $(1-5)^{\mathrm{a}}$} & \multirow{2}{*}{\multicolumn{4}{|c|}{ RTs $(\mathrm{msec})^{\mathrm{b}}$}} \\
\hline & \multirow{2}{*}{\multicolumn{2}{|c|}{$\begin{array}{l}\text { Youths With } \\
\text { a History } \\
\text { of Caregiver } \\
\text { Deprivation } \\
\text { and Emotional } \\
\text { Neglect } \\
(N=11)\end{array}$}} & \multirow{2}{*}{\multicolumn{2}{|c|}{$\begin{array}{c}\text { Comparison } \\
\text { Youths } \\
(N=19)\end{array}$}} & & & & \\
\hline & & & & & \multicolumn{2}{|c|}{$\begin{array}{c}\text { Youths With } \\
\text { a History of } \\
\text { Caregiver } \\
\text { Deprivation and } \\
\text { Emotional Neglect } \\
(N=11)\end{array}$} & \multicolumn{2}{|c|}{$\begin{array}{c}\text { Comparison } \\
\text { Youths } \\
(N=19)\end{array}$} \\
\hline & $M$ & $S D$ & $M$ & $S D$ & $M$ & $S D$ & $M$ & $S D$ \\
\hline \multicolumn{9}{|c|}{ How afraid are you? } \\
\hline Angry & 2.64 & 1.14 & 2.33 & 1.13 & $1,618.44$ & 414.53 & $2,124.72$ & 588.49 \\
\hline Fearful & 1.95 & 0.79 & 1.87 & 0.92 & $1,736.00$ & 408.77 & $1,924.05$ & 476.23 \\
\hline Нарру & 1.19 & 0.48 & 1.09 & 0.18 & $1,455.14$ & 372.52 & $1,438.09$ & 322.79 \\
\hline Neutral & 1.53 & 0.40 & 1.45 & 0.56 & $1,684.32$ & 410.41 & $1,759.55$ & 391.55 \\
\hline \multicolumn{9}{|c|}{ How hostile is the face? } \\
\hline Angry & 3.46 & 1.52 & 3.10 & 1.21 & $1,561.88$ & 378.10 & $2,066.38$ & 445.84 \\
\hline Fearful & 2.36 & 1.11 & 2.02 & 0.94 & $1,965.03$ & 473.47 & $2,135.19$ & 518.00 \\
\hline Happy & 1.33 & 0.62 & 1.06 & 0.14 & $1,377.36$ & 275.96 & $1,583.35$ & 360.71 \\
\hline Neutral & 1.94 & 0.66 & 1.68 & 0.54 & $1,671.45$ & 269.57 & $1,949.15$ & 370.92 \\
\hline \multicolumn{9}{|c|}{ How wide is the nose? } \\
\hline Angry & 2.52 & 0.67 & 2.58 & 0.72 & $1,670.96$ & 337.17 & $2,155.23$ & 484.80 \\
\hline Fearful & 2.21 & 0.61 & 2.05 & 0.59 & $1,723.08$ & 279.14 & $2,034.69$ & 405.99 \\
\hline Happy & 2.58 & 0.63 & 2.49 & 0.53 & $1,776.96$ & 357.97 & $2,218.33$ & 405.40 \\
\hline Neutral & 2.13 & 0.50 & 2.10 & 0.57 & $1,711.88$ & 300.61 & $1,976.40$ & 407.82 \\
\hline
\end{tabular}

aEach face type was rated from 1 to 5 , with $1=$ low level and $5=$ high level of fear, hostility, or nose width. ${ }^{\mathrm{b}}$ Longer $\mathrm{RT}=$ more time taken to rate the face.

With respect to RTs, there was a significant main effect of group $[F(1,28)=5.75, p=.023]$, subsumed by a significant two-way interaction of group $\times$ emotional faces $[F(3,84)=4.87, p=.004]$. Post hoc analyses revealed that caregiver-deprived and emotionally neglected youths showed faster RTs than did comparison youths for angry faces $\left(p<.05, d^{\prime}=1.13\right.$; Figure 1$)$. The ANOVA also revealed significant main effects of emotional faces $[F(3,84)=13.94, p=.0001]$ and attention states $[F(2,56)=8.69, p=.001]$, which were subsumed by a significant two-way interaction of emotional faces $X$ attention states $[F(6,168)=12.06, p=.001]$. Post hoc analyses showed that fearful faces required the longest RT to make the "hostile" ratings, but the shortest RT to make the "afraid" ratings (all $p \mathrm{~s}<.05$ ). Happy faces required the longest RT to make the "nose width" ratings, but the shortest RT to make the "afraid" and "hostile" ratings (all $p$ s $<.05$; Table 2).

Subjective rating scores and RT measures were reanalyzed using ANOVAs after removing the two caregiver-

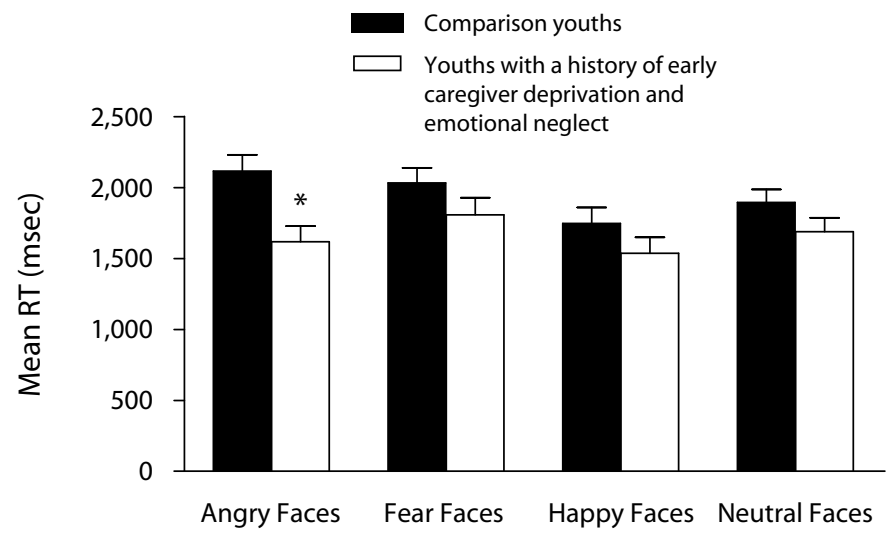

Type of Emotional Faces

Figure 1. Mean reaction times (RTs) for youths with a history of caregiver deprivation and emotional neglect $(N=11)$ and comparison youths $(N=19)$, according to face emotion. ${ }^{*} p<.05$. 
deprived and emotionally neglected youths who had anxiety disorders, and all of the results reported previously remained significant.

In summary, youths' subjective ratings were not moderated by group, but RTs were significantly faster among caregiver-deprived and emotionally neglected youths than they were among comparison youths when rating angry faces. This effect of group on RTs was not modulated by the different attention states.

\section{Imaging Data}

We first investigated whether caregiver-deprived and emotionally neglected youths would differ from comparison youths with regard to amygdala, anterior hippocampus, and posterior hippocampus activation in response to fearful versus neutral faces, in the How afraid are you? attention state. Results showed significantly greater activation in the left $\left[t(28)=3.17, p=.035, d^{\prime}=1.20\right]$ and right $\left[t(28)=3.35, p=.026, d^{\prime}=1.27\right]$ amygdalae and left anterior hippocampus $\left[t(28)=3.62, p=.016, d^{\prime}=\right.$ $1.37]$ in caregiver-deprived and emotionally neglected youths versus in comparison youths (see Table 3 for coordinates; Figures 2A and 3A). Follow-up ANOVAs using SPSS 14.0 on the BOLD responses in the identified peak suprathreshold voxels (see Table 3 ) revealed significant two-way interactions of group $\times$ emotional faces in the left amygdala $[F(3,84)=5.00, p=.003]$ and left anterior hippocampus $[F(1.8,50.48)=7.18, p=.002]$. These interactions reflected significantly greater left amygdala $\left(p=.039, d^{\prime}=0.76\right.$; Figure 2B) and left anterior hippocampus $\left(p=.042, d^{\prime}=0.74\right.$; Figure $3 \mathrm{~B}$ ] activation to fearful faces in youths with a history of caregiver deprivation and emotional neglect, relative to that in comparison youths. No significant main effects or interactions were found for the right amygdala (all $F \mathrm{~s}<1.5$, all $p \mathrm{~s}>.1$ ).

Second, we investigated whether youths with a history of caregiver deprivation and emotional neglect would differ from comparison youths with regards to amygdala, anterior hippocampus, and posterior hippocampus activation in response to angry versus neutral faces, in the How afraid are you? attention state. Results show significantly greater activation in the left amygdala $[t(28)=2.84, p=$
$.05, d^{\prime}=1.07$; Table 3; Figure 4A] and left anterior hippocampus $\left[t(28)=2.94, p=.046, d^{\prime}=1.11\right.$; Table 3] in caregiver-deprived and emotionally neglected youths versus in comparison youths. Follow-up ANOVAs using SPSS 14.0 on the BOLD responses in the identified peak suprathreshold voxels (see Table 3 ) revealed a significant two-way interaction of group $\times$ emotional faces in the left amygdala $[F(3,84)=3.01, p=.034]$. This interaction reflected significantly greater left amygdala activation ( $p=$ $.05, d^{\prime}=0.70$; Figure 4B) to fearful faces in youths with a history of caregiver deprivation and emotional neglect relative to that in comparison youths. No significant main effects or interactions were found for the left anterior hippocampus (all $F \mathrm{~s}<2.7$, all $p \mathrm{~s}>.05$ ).

SPM analyses, as well as follow-up ANOVAs using activation values at the identified peak suprathreshold voxels, were rerun for the contrast fearful versus neutral faces after removing the 2 caregiver-deprived and emotionally neglected youths who had anxiety disorders. SPM findings remained significant, with greater left amygdala $[t(26)=$ $\left.3.37, p=.025, d^{\prime}=1.32\right]$, right amygdala $[t(26)=3.34$, $\left.p=.028, d^{\prime}=1.31\right]$, and left anterior hippocampus $\left[t(26)=3.39, p=.026, d^{\prime}=1.33\right]$ activation observed in youths with a history of caregiver deprivation and emotional neglect relative to that in comparison youths. Follow-up ANOVAs showed that the group $\times$ emotional faces interaction remained significant in the left amygdala $[F(3,78)=3.08, p=.032]$, with caregiver-deprived and emotionally neglected youths persistently showing significantly greater activation to fearful faces than did comparison youths $\left(p=.019, d^{\prime}=0.87\right)$. Removing the 2 participants with diagnoses also led caregiver-deprived and emotionally neglected youths to show significantly greater activation to angry faces than did comparison youths $\left(p=.034, d^{\prime}=0.81\right)$. However, despite a significant group $\times$ emotional faces interaction in the left anterior hippocampus $[F(1.7,45.04)=6.51, p=.005]$, simple effects revealed that previous group differences for fearful faces fell to a trend level $\left(p=.087 ; d^{\prime}=0.63\right)$. This may be attributable to insufficient power. No other group differences were observed for the left anterior hippocampus $(p s>.1)$. No significant main effects or inter-

Table 3

Peak Voxels for the Contrast Caregiver-Deprived and Emotionally Neglected Youths-Comparison Youths, for Fearful Versus Neutral Faces and Angry Versus Neutral Faces in the How Afraid Are You? Attention State for the Amygdalae, Anterior Hippocampi, and Posterior Hippocampi

\begin{tabular}{|c|c|c|c|c|c|c|c|c|c|c|c|c|}
\hline \multirow[b]{2}{*}{ Primary Analysis (SPM) } & \multicolumn{6}{|c|}{$\begin{array}{l}\text { Fearful Faces Versus Neutral Faces } \\
\text { in the Afraid Attention State }\end{array}$} & \multicolumn{6}{|c|}{$\begin{array}{l}\text { Angry Faces Versus Neutral Faces } \\
\text { in the Afraid Attention State }\end{array}$} \\
\hline & $\begin{array}{c}\text { Cluster } \\
\text { Size } \\
\text { (voxels) }\end{array}$ & $x$ & $y$ & $z$ & $t(28)$ & $p^{\mathrm{a}}$ & $\begin{array}{c}\text { Cluster } \\
\text { Size } \\
\text { (voxels) }\end{array}$ & $x$ & $y$ & $z$ & $t(28)$ & $p^{\mathrm{a}}$ \\
\hline Left amygdala & 48 & -14 & -2 & -22 & 3.17 & .035 & 33 & -22 & -2 & -22 & 2.84 & .050 \\
\hline Right amygdala & 91 & 24 & 0 & -24 & 3.35 & .026 & 60 & 32 & 2 & -28 & 2.64 & .074 \\
\hline Left anterior hippocampus & 129 & -12 & -8 & -18 & 3.62 & .016 & 146 & -22 & -4 & -20 & 2.94 & .046 \\
\hline Right anterior hippocampus & 16 & 26 & -2 & -24 & 2.96 & .061 & 12 & 26 & -2 & -26 & 2.40 & .125 \\
\hline Left posterior hippocampus & 51 & -28 & -26 & -12 & 2.92 & .080 & 115 & -32 & -22 & -16 & 1.99 & .291 \\
\hline Right posterior hippocampus & 22 & 20 & -26 & -12 & 2.77 & .107 & 7 & 22 & -24 & -14 & 2.08 & .272 \\
\hline
\end{tabular}

Note-Coordinates $(\mathrm{mm})$ are reported in Montreal Neurological Institute space. aAll voxelwise $t$ values are significant at $\alpha=.05$, based on a small volume correction for multiple comparisons in each region. For youths with a history of caregiver deprivation and emotional neglect, $N=11$ 
A
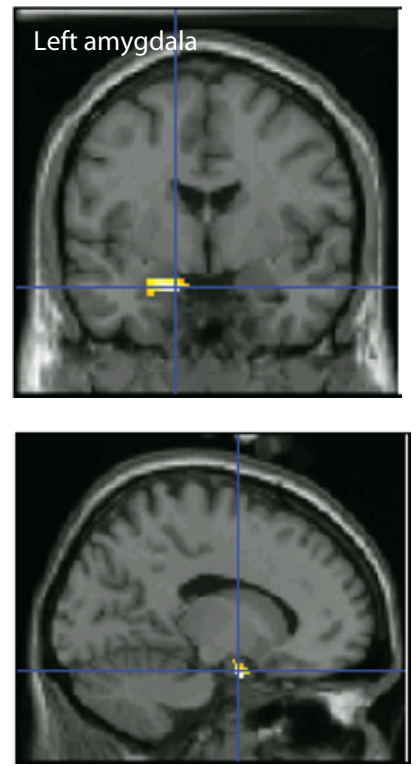

B

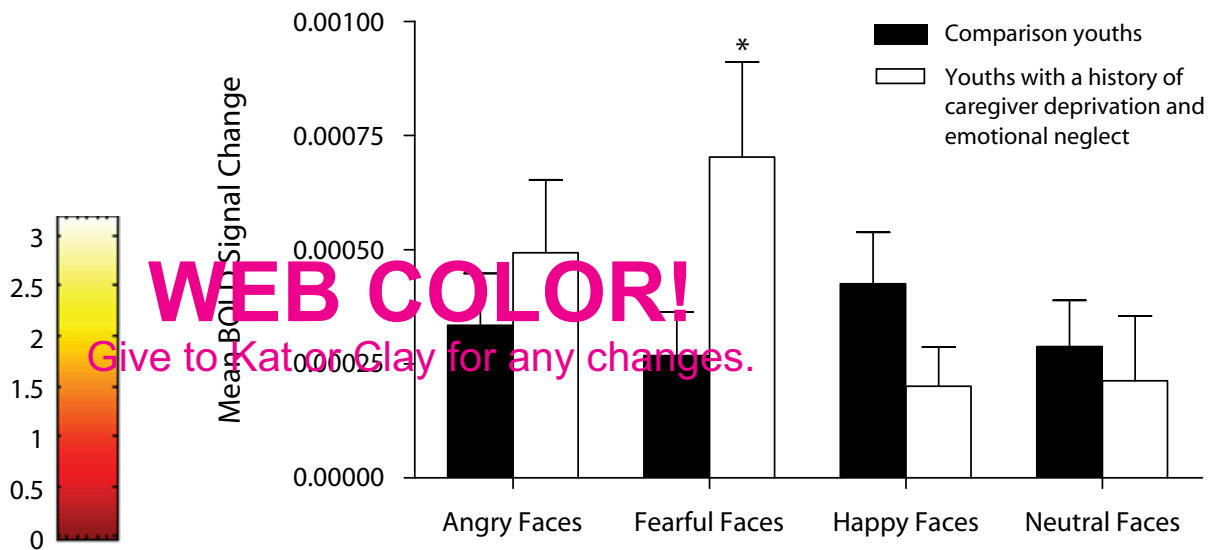

Type of Emotional Faces

Figure 2. (A) Left amygdala activation among youths with a history of caregiver deprivation and emotional neglect $(N=11)$ and comparison youths $(N=19)$ in the contrast fearful versus neutral faces in the afraid attention state. (B) Mean BOLD signal changes extracted at the identified peak voxel during the viewing of fearful faces versus neutral faces (across all attention states). ${ }^{*} p=.039$.

actions were found for the right amygdala (all $F_{\mathrm{S}}<1.4$; all $p \mathrm{~s}>.1)$.

Similar analyses were rerun for the contrast angry versus neutral faces, after removing the 2 youths in the experimental group who had anxiety disorders. SPM findings remained significant, with greater left amygdala $[t(26)=$ $\left.3.83, p=.007, d^{\prime}=1.50\right]$ and left anterior hippocampus $\left[t(26)=4.77, p=.001, d^{\prime}=1.87\right]$ activation observed in youths with a history of caregiver deprivation and emotional neglect relative to that in comparison youths. Follow-up
A
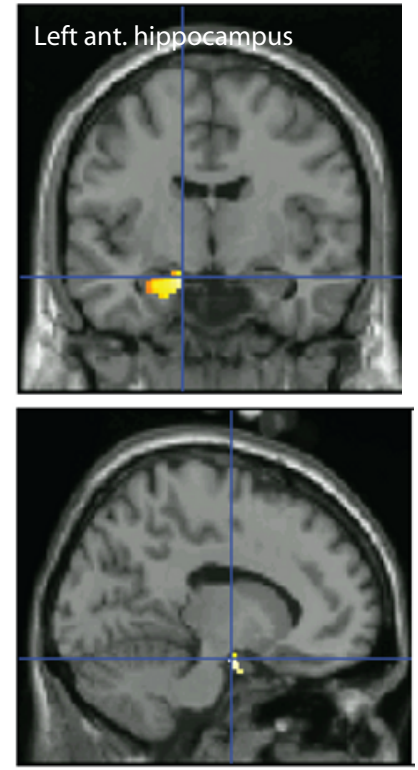

B

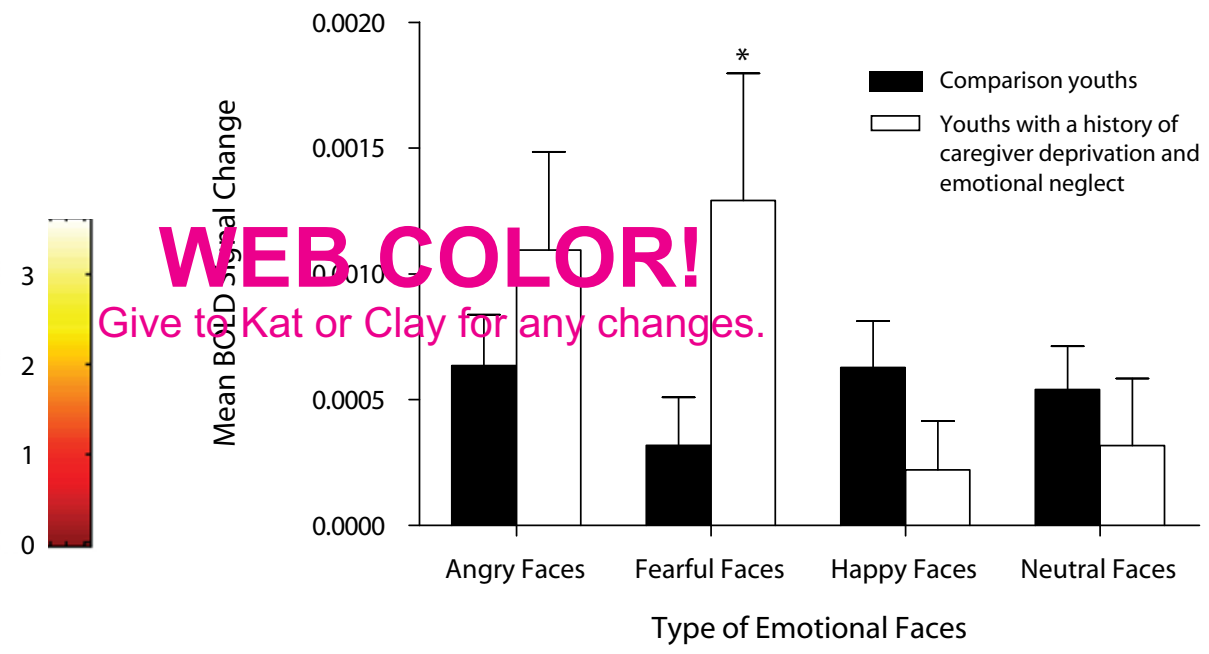

Figure 3. (A) Left anterior hippocampus activation among youths with a history of caregiver deprivation and emotional neglect $(N=11)$ and comparison youths $(N=19)$ in the contrast fearful versus neutral faces in the afraid attention state. (B) Mean BOLD signal changes extracted at the identified peak voxel during the viewing of fearful faces versus neutral faces (across all attention states). $\quad{ }^{*} p=.042$. 
A
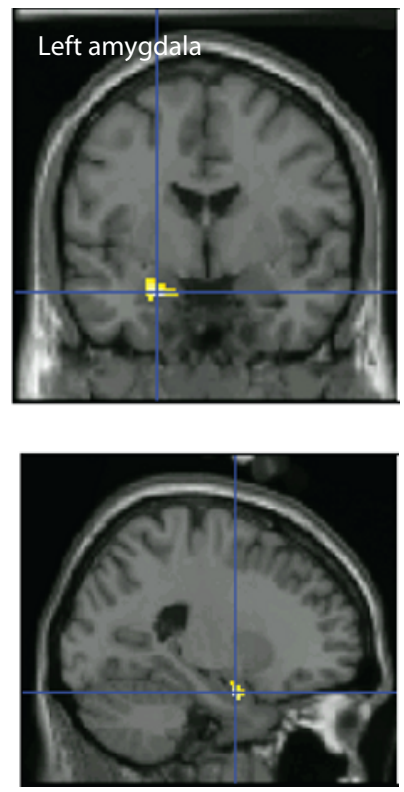

B

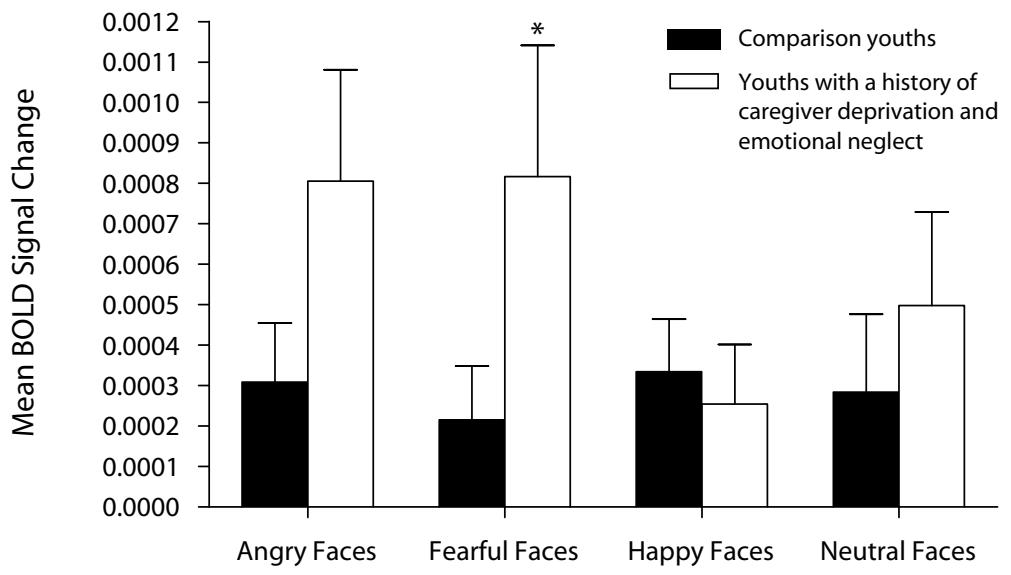

Type of Emotional Faces

Figure 4. (A) Left amygdala activation among youths with a history of caregiver deprivation and emotional neglect $(N=11)$ and comparison youths $(N=19)$ in the contrast angry versus neutral faces in the afraid attention state. (B) Mean BOLD signal changes extracted at the identified peak voxel during the viewing of fearful faces versus neutral faces (across all attention states). ${ }^{*} p=.05$.

ANOVAs showed that the group $\times$ emotional faces interaction remained significant in the left amygdala $[F(3,78)=$ $2.84, p=.043]$, with caregiver-deprived and emotionally neglected youths persistently showing significantly greater activation to fearful faces than did comparison youths $(p=$ $\left..041, d^{\prime}=0.76\right)$. Removing the 2 participants with diagnoses also led caregiver-deprived and emotionally neglected youths to show greater activation to angry faces than did comparison youths ( $\left.p=.042, d^{\prime}=0.80\right)$. In addition, the group $X$ attention states interaction became significant $[F(3,78)=3.08, p=.032]$, with greater left amygdala activation observed in caregiver-deprived and emotionally neglected youths versus comparison youths during the "subjective feelings of fear" attention state (i.e., the How afraid are you? attention state; Figures 5A and 5B). No significant main effects or interactions were found for the left anterior hippocampus (all $F \mathrm{~s}<2.7$, all $p \mathrm{~s}>.05$ ).

Given the group differences in RTs to angry faces, we examined the influence of this variable on brain imaging findings. Mean RT to angry faces across all attention states was used as the covariate. The RT to angry faces had no significant effects on emotional faces, attention states, or emotional faces $\times$ attention states modulation of left amygdala or left anterior hippocampus activity in each group separately, or in the whole group, in the contrast fearful versus neutral faces. Similar findings for the left amygdala were observed in the contrast angry versus neutral faces. In addition, all group effects on the regional activations identified previously remained significant, except for left anterior hippocampus activation in the contrast fearful versus neutral faces $(N=11$ in the experimental group). Since the RT in itself did not modulate brain activation, its effect on group differences in the left anterior hippocampus activation reflected the differences in group means rather than a direct effect of RT on brain activation.

In summary, left amygdala and left anterior hippocampus activation in the contrast fearful versus neutral faces, and left amygdala activation in the contrast angry versus neutral faces, were moderated by group and emotional faces. Indeed, in both contrasts, youths with a history of caregiver deprivation and emotional neglect showed significantly greater neural responses in these structures during the viewing of fearful faces than did comparison youths. Attention states did not moderate left amygdala or left anterior hippocampus activation. However, when the 2 participants with anxiety disorders were removed from the experimental group, findings varied slightly. Left amygdala activation for both contrasts was modulated by group and emotional faces, with caregiver-deprived and emotionally neglected youths showing significantly greater activation to fearful and angry faces. In the angry versus neutral faces contrast, left amygdala modulation was also influenced by attention, with caregiver-deprived and emotionally neglected youths showing, relative to comparison youths, significantly greater activation during the "subjective feelings of fear" attention state.

\section{Correlations Between Demographic, As Well As Caregiver and Adoption-Related Variables, and Regional Activations}

Age. Correlation analyses of age with the peak activations of the left amygdala and left anterior hippocampus for the contrasts afraid-fearful faces versus afraid-neutral 
A
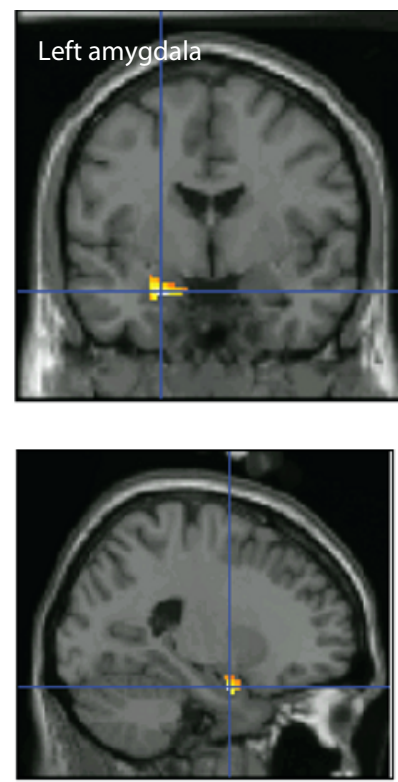

B

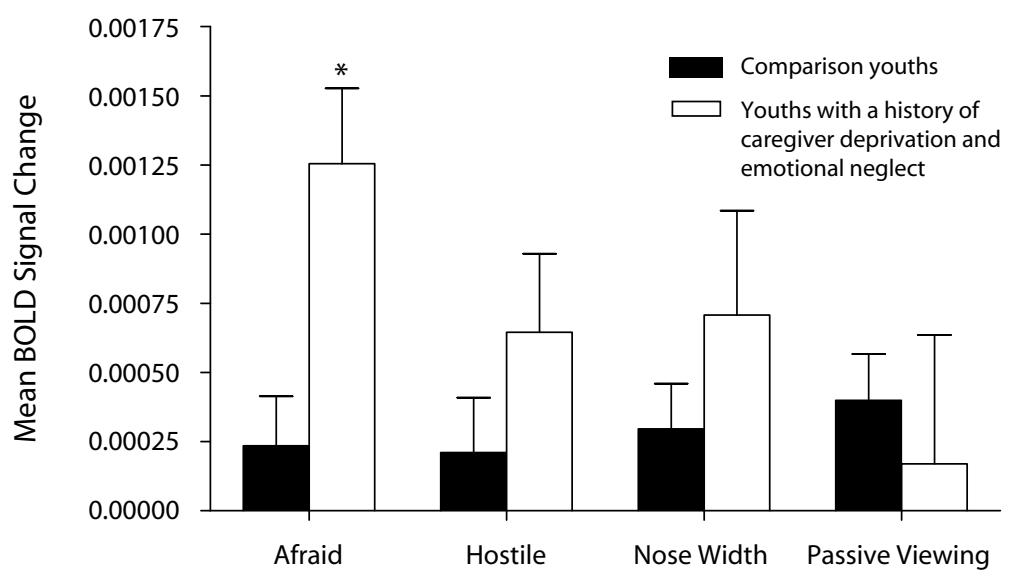

Attention States

Figure 5. (A) Left amygdala activation among youths with a history of caregiver deprivation and emotional neglect after removing the 2 participants with an anxiety disorder $(n=9)$, and comparison youths $(N=19)$, in the contrast angry versus neutral faces in the afraid attention state. (B) Mean BOLD signal changes extracted at the identified peak voxel during the "How afraid are you?" attention state. ${ }^{*} p<.05$.

faces and afraid-angry faces versus afraid-neutral faces were not significant either in youths with a history of caregiver deprivation and emotional neglect $(n=9$ or $N=11)$ or in the comparison group (all $p \mathrm{~s}>.1$ ).

Characteristics of caregiver deprivation and adoption. Similarly, correlation analyses were conducted between caregiver deprivation and adoption-related variables, and the peak activations of the left amygdala and left anterior hippocampus. In the contrast angry versus neutral faces, left amygdala activation to fearful faces in the caregiver-deprived and emotionally neglected youths was positively correlated with the number of placements $(N=11, r=.81, p=.002 ; n=9, r=.82, p=.006)$ and negatively correlated with the time (in months) spent in the adoptive family since being placed in the family $(N=$ $11, r=-.78, p=.005 ; n=9, r=-.082, p=.007)$.

\section{DISCUSSION}

In the present preliminary study, youths with a history of caregiver deprivation and emotional neglect had significantly greater medial temporal lobe activation during the processing of threatening cues. Indeed, when considering all of the participants in the experimental group $(N=11)$, greater activation in the left amygdala (during the contrasts fearful versus neutral faces and angry versus neutral faces) and left anterior hippocampus (during the contrast fearful versus neutral faces) in response to fearful faces was observed in caregiver-deprived and emotionally neglected youths, relative to that in a comparison group. These exaggerated responses in the experimental group were seen across all attention states and were therefore not specific to the fear-focused attention state. However, these results were slightly modified after removing the 2 participants with anxiety disorders from the experimental group. In this context, caregiver-deprived and emotionally neglected youths had significantly greater left amygdala activation (in the fearful versus neutral faces and angry versus neutral faces contrasts) during the processing of both fearful and angry faces. The subjective fear attention state (How afraid are you?) modulated this exaggerated left amygdala activation during the angry versus neutral faces contrast only. Interestingly, regardless of whether the diagnosed youths were included in the experimental group, left amygdala activation during the angry versus neutral faces contrast was positively related to the number of placements in foster care or institutionalization, and was negatively related to the time spent in the adoptive family. This suggests that the left amygdala may have a particular sensitivity to specific caregiver deprivation and adoption-related characteristics.

Regarding behavioral performance, group differences did not emerge in youths' ratings of pictures. However, group differences for RTs were present. Youths who had experienced caregiver deprivation and emotional neglect rated angry faces significantly faster than did comparison youths. Finally, subjective ratings and RTs varied as a function of attention states and types of emotional faces in both groups. As was expected, threatening faces were rated as more negative than were happy faces. Additionally, participants were faster in determining that happy faces were not hostile or frightening, but they were slower 
in evaluating the nose wideness of these faces. A contrary pattern was observed for threatening faces.

Altogether, these preliminary findings suggest that youths with a history of caregiver deprivation and emotional neglect are behaviorally more sensitive to threatening facial expressions, and that enhanced medial temporal lobe activation may serve as a neural underpinning of this sensitivity.

Our RT findings in caregiver-deprived and emotionally neglected youths are consistent with those in previous research indicating that youths who were neglected and abused - regardless of whether they had a history of foster care or institutionalization-are faster than comparison youths in identifying threatening faces (Masten et al., 2008; Pollak \& Tolley-Schell, 2003). This heightened sensitivity to threatening faces might serve to protect caregiver-deprived as well as neglected and abused youths against additional aversive situations by rapidly identifying negative cues. Regarding the ratings, however, no between-group differences emerged in characterizing angry, fearful, happy, or neutral faces as hostile, frightening, or having a wide nose. It is unlikely that a lack of sensitivity in the task may account for these results. Indeed, our task was sensitive enough to detect between-group differences in brain activation for specific types of emotional faces (fearful and angry faces), and the effect size for these group differences was medium to large (all effect sizes $>0.7$ ). Most likely, true differences in the rating of emotional faces between caregiver-deprived and emotionally neglected youths and the comparison group may not have been detected due to insufficient power, since the sample size of the experimental group was low.

Regarding the imaging findings of the present preliminary study, the left amygdala and left anterior hippocampus results that were observed in the caregiver-deprived and emotionally neglected youths parallel previous findings in postinstitutionalized adoptees demonstrating alterations in medial temporal lobe regions. Chugani et al. (2001) reported reduced left medial temporal lobe metabolism in postinstitutionalized Romanian youths as compared with that of epileptic youths and healthy adults. Greater amygdala volume (Mehta et al., 2009) and reduced integrity of the uncinate fasciculus - white matter tracts connecting the anterior temporal lobe to the inferior frontal lobe (Eluvathingal et al., 2006) — were also shown in youths who were adopted from Eastern European orphanages as compared with control youths. Although the link between the uncinate fasiculus and proximal gray matter structures such as the amygdala is not clear, this result, taken together with our findings and those of Mehta et al. and Chugani et al., strengthens the suggestion of medial temporal lobe abnormalities in caregiver-deprived youths.

Our medial temporal lobe preliminary findings also parallel data reported by studies investigating the medial temporal lobe in maltreated youths and adults. In these studies, experiences of foster care or institutionalization for participants were not reported. However, anxiety disorders were observed in all of the participants in these studies. Reduced medial temporal lobe volume (De Bellis et al., 2002) and altered hippocampal volume (reduced volume, Carrion et al., 2007; increased volume, Tupler \& De Bellis, 2006) were reported in anxious youths with a history of neglect and abuse. In adults, functional neuroimaging findings reported abnormal decreases in hippocampal activation and abnormal increases in amygdala activation during the processing of threat cues in patients with anxiety disorders related to early childhood neglect and abuse (Bremner, 2007; Bremner et al., 1999; Bremner et al., 2005; Bremner, Vythilingam, Vermetten, Southwick, McGlashan, Nazeer, et al., 2003; Bremner, Vythilingam, Vermetten, Southwick, McGlashan, Staib, et al., 2003).

Of note, the medial temporal lobe perturbations observed in the present study are in the opposite direction of those found by Chugani et al. (2001) in postinstitutionalized Romanian children adopted by American families. Comparing our study to that of Chugani et al. is, however, complicated by the number of major methodological differences existing between the studies. First, and most importantly, we examined brain activity during the processing of cognitive emotional cues, whereas Chugani et al. studied brain activity at rest. Thus, the neural activity measured by both studies was of different origins, making a comparison of brain function between investigations difficult. Second, we used fMRI with a temporal resolution of $4 \mathrm{sec}$, whereas Chugani et al. used PET with a temporal resolution of $20 \mathrm{~min}$. These differences in imaging technology, as well as the lower image resolution in Chugani et al.'s study, may have complicated comparisons with our findings. The age of participants also differed between studies. Our youths with a history of caregiver deprivation and emotional neglect were 13.7 years old on average, whereas Chugani et al.'s postinstitutionalized children were 8.8 years old on average. Thus, divergent findings between studies could be attributable to the differences in neural activity that are shown to occur with increasing age (Monk et al., 2003). Finally, the comparison groups were different. Although we used a healthy comparison group, Chugani et al. used children with seizure disorders as a control group. The use of a patient control group, rather than of a healthy control group, may have affected the conclusions drawn regarding brain function in the postinstitutionalized adoptees investigated by Chugani et al.

Other discrepancies between our and others' findings are also observed. In the adult maltreatment studies, reduced hippocampal activity during the processing of threat cues was observed in patients with childhood maltreatmentrelated PTSD (Bremner et al., 1999; Bremner, Vythilingam, Vermetten, Southwick, McGlashan, Nazeer, et al., 2003; Bremner, Vythilingam, Vermetten, Southwick, McGlashan, Staib, et al., 2003). In contrast, we report exaggerated hippocampal activation in youths with a history of caregiver deprivation and emotional neglect. Here again, methodological differences may account for these conflicting findings. Besides imaging techniques (fMRI vs. PET), the age as well as the type of tasks used (emotional attention task vs. emotional memory task) and the psychiatric profile (18\% of the present sample suffered from anxiety versus $100 \%$ of the adult samples carried a diagnosis of PTSD) are critical features that differ between studies. These different features may have influenced the functioning of the hippocampus in unique ways. However, it is also possible that, 
with time, exaggerated activity within the hippocampus reverses to abnormally low activity as a result of excitotoxicity from sustained glutamate synaptic excess (Armanini, Hutchins, Stein, \& Sapolsky, 1990; Zhou \& Baudry, 2006). This hypothesis is particularly interesting, since it may further suggest that the individuals who develop PTSD are those whose hippocampus is particularly sensitive to longterm effects of glutamate excess.

Despite these discrepancies, the consistently reported locus of atypical activity - that is, the medial temporal lobe - strongly suggests that caregiver deprivation as well as neglect and abuse influence the functioning of specific brain regions. Whether these adverse experiences have an impact on the functional integrity of the medial temporal lobe only, or whether they modulate a distributed neural network that includes the medial temporal lobe, needs to be further assessed. As such, the data reported by Eluvathingal et al. (2006) on structural alterations in the neural pathway connecting the frontal lobe, which are implicated in the inhibitory control of subcortical limbic structures (i.e., the amygdala, the hippocampus), and the anterior temporal lobe in youths adopted from Eastern European orphanages are particularly relevant to this issue. As such, these results hint at the presence of a possible deficit in frontal inhibitory modulation of subcortical limbic structures in caregiver-deprived and neglected populations. This could underlie the enhanced left amygdala and left anterior hippocampus activation observed in the present study.

Another interesting finding of the present preliminary study is that exaggerated activation was reported in the amygdala and anterior hippocampus of the left hemisphere, but not of the right hemisphere. Indeed, although the SPM analysis revealed group differences in the right amygdala for the contrast fearful versus neutral faces, follow-up ANOVAs performed on the BOLD data extracted at the most significant peak voxel did not confirm these group differences (see the Results section). In addition, no group differences in right amygdala activation were observed in the other contrast (angry vs. neutral faces). Interestingly, left medial temporal lobe structures were recently shown to be more activated than right medial temporal lobe structures during the processing of negative emotional stimuli, especially facial cues (Baas, Aleman, \& Kahn, 2004; FusarPoli et al., 2009). The left medial temporal lobe is reported to be closely implicated in more conscious, specific, and sustained emotional coding, such as extensive processing of local, fine-grained aspects of emotional cues. The right medial temporal lobe, on the other hand, is found to be mostly implicated in automatic, fast, and short analysis of global, holistic aspects of emotional stimuli (Baas et al., 2004; Fusar-Poli et al., 2009; Kim et al., 2007). Since the task in the present study necessitated more mental analysis of the detailed emotional facial expressions, it logically solicited the more specific and local emotional processing functions of the left amygdala. Left and right posterior hippocampi are other regions that were unsolicited by the emotional task of the present preliminary study. This is not surprising, since the posterior hippocampi are implicated in providing contextual memories to emotional cues, whereas the anterior hippocampi and amygdalae are implicated in the processing of emotional cues per se (Bannerman et al., 2004; Dolcos et al., 2004; LeDoux, 2000).

In addition, the left anterior hippocampus, as was demonstrated by the follow-up ANOVAs on the extracted BOLD data, was activated in response to fearful faces, but not to angry faces. This finding parallels previous results reporting that the hippocampus is not central to the coding of angry facial features. This facial emotional expression activates most consistently the medial prefrontal cortex, ventromedial prefrontal cortex, lateral prefrontal cortex, anterior and posterior cingulate cortices, amygdala, and thalamus (Denson, Pedersen, Ronquillo, \& Nandy, 2008; Murphy, Nimmo-Smith, \& Lawrence, 2003; Park et al., 2008; Phan, Wager, Taylor, \& Liberzon, 2002). Rather, the hippocampus is thought to be implicated in processing the rumination of angry feelings (Denson et al., 2008). Angry faces also failed to trigger significant amygdala response when all of the participants in the experimental group were considered $(N=11)$. Indeed, a closer examination of Figures 2 and 4 shows that, similar to fearful faces, angry faces activated the amygdala more than did happy or neutral faces. But this activation did not reach significance. However, when the two youths with anxiety disorders were removed from the experimental group, the amygdala response became significant not only to fearful faces but to angry faces as well, relative to the response to happy and neutral faces. This suggests that removing the influence of anxiety symptoms may have facilitated amygdala response to angry faces. This is surprising, since the amygdala is shown to be particularly sensitive to angry faces in youths with anxiety disorders (Monk et al., 2006; Monk et al., 2008). It is thus possible that this unexpected opposite direction in findings is attributable to statistical variance in the experimental group. Investigating a larger sample may help determine more specifically the influence of angry faces on amygdala function in caregiverdeprived and emotionally neglected youths.

Another point that needs to be addressed is the influence of the subjective feelings of fear on brain function. Contrary to what was expected, there was no influence of subjective feelings of fear on the left amygdala and left anterior hippocampus activation in caregiver-deprived and emotionally neglected youths, when all of the participants in the experimental group were considered $(N=$ 11 ). This could be attributable to a pervasive effect of the enhanced medial temporal lobe function across attention states, which may reflect a more profound functional impairment of this structure. However, when the 2 participants with anxiety disorders were removed from the experimental sample $(n=9)$, subjective feelings of fear did have a modulatory influence on left amygdala function (see Figure 5). Such findings are in contradiction with studies reporting a modulatory influence of subjective feelings of fear on amygdala function in youths with, or at risk for, anxiety disorders in particular (McClure et al., 2007; Pérez-Edgar et al., 2007). Studying a higher number of participants and creating subsamples of participants with and without anxiety disorders would allow us to determine more specifically whether it is the anxiety symptoms or the subjective feelings of fear that influence 
medial temporal lobe function in caregiver-deprived and emotionally neglected youths.

Finally, greater left amygdala activation was positively related to the number of placements in foster care or an institution, suggesting higher amygdala reactivity with a higher number of placements. Repeated moves of youths from one living environment to another are thought to enhance stress and to prevent the establishment of a secure attachment relationship with caregivers, leading to impaired development. In line with this notion, greater emotional, relational, or behavioral problems have been observed in caregiver-deprived youths with higher numbers of placements (American Academy of Pediatrics, 2000; Halfon, Mendonca, \& Berkowitz, 1995; Nickman et al., 2005). Since the amygdala is related to emotional, relational, and behavioral function (Adolphs, 2003), abnormalities in amygdala activity could underlie these difficulties in caregiver-deprived youths. Left amygdala activity was also negatively related to the time spent in the adoptive family - a result that parallels previous findings in postinstitutional Romanian adoptees showing performance improvement on emotion or cognitive tasks as the time in adoptive homes increases (Rutter et al., 2004; Wismer-Fries \& Pollak, 2004). These findings, taken together with our results, suggest that positive developmental effects may be observed in youths following adoption in a caring family environment.

However, no influence of age at placement (in foster care/institution), age at adoption, or length of time spent in foster care/institution was observed in the present preliminary study. This is surprising, since these variables were found to influence emotional and cognitive processing in postinstitutionalized or foster care youths (see, e.g., Kreppner et al., 2007; Mehta et al., 2009; Pears \& Fisher, 2005a, 2005b; Rutter et al., 2004; Wismer-Fries \& Pollak, 2004). Two factors may contribute to this lack of correlations. First, it is possible that these variables have an impact on brain regions other than the medial temporal lobe - for example, the prefrontal cortex. Second, we studied a small sample of caregiver-deprived and emotionally neglected youths with a wide range of age at placement, age at adoption, and length of time spent in foster care/ institution (e.g., see the Method section). Investigating a larger sample would allow us to make subsamples and to determine more precisely the influence of these variables on medial temporal lobe function.

Thus, the neuroimaging findings of the present preliminary study are consistent with the hypothesis that caregiver deprivation and emotional neglect may affect the development of neural systems modulating emotional processes. Importantly, even though some of our findings suggest positive effects after the restoration of normal family rearing, abnormalities in medial temporal lobe function seem to persist for an important amount of time after youths are placed in their adoptive homes. Indeed, the present findings suggest that even the stable family environment in which the caregiver-deprived and emotionally neglected youths have been reared for an average of 8 years ( 97 months) did not completely override the influence of caregiver deprivation and emotional neglect. This suggests that the organizational effects of caregiver deprivation and emotional neglect on the immature brain may not be fully reversible.

In support of this idea, animal studies report long-lasting detrimental effects of early-life caregiver deprivation on brain development and function. For instance, rodent offspring that are exposed to early maternal separation show alterations in the structure and function of the amygdala and hippocampus when tested at puberty or as adults (Sanchez, 2006; Sanchez et al., 2001; Teicher et al., 2002). In humans, caregiver-deprived and maltreated youths show dysfunctions in cognitive and emotional functions dependent on the medial temporal lobe years after placement in adoptive homes (e.g., Pears \& Fisher, 2005a, 2005b; Pollak, 2005; Rutter et al., 2004). Additionally, structural and functional alterations of the medial temporal lobe in postinstitutionalized or maltreated pre-adolescents and adolescents, as well as in adults with a history of childhood maltreatment, suggest that early-life adversities are associated with aberrant brain regulation and function many years after exposure to the adverse environment (see, e.g., Bremner, 2007; Chugani et al., 2001; Eluvathingal et al., 2006; Gunnar et al., 2000; Mehta et al., 2009; Rutter et al., 2004; Tupler \& De Bellis, 2006). Hence, long-lasting influences of caregiver deprivation as well as maltreatment on brain development probably underlie the enhanced sensitivity to threat cues and elevated risk for anxiety and depression disorders that are documented in caregiver-deprived and maltreated youths.

Interestingly, only 2 youths with a history of caregiver deprivation and emotional neglect in the present preliminary study presented anxiety disorders, and no group differences were observed on scales measuring anxiety (SCARED) and depression (CDI). Although insufficient power could explain this negative finding, the possibility that psychiatric disorders could emerge later in the caregiver-deprived and emotionally neglected youths needs to be considered. Longitudinal studies would need to be conducted to reliably address this question.

\section{Limitations and Recommendations}

Our preliminary findings should be considered in light of study limitations. First, the results are based on a relatively small sample size. Although statistical power was adequate to detect group differences in left amygdala and left anterior hippocampus activation, a restricted sample size may have limited our ability to (1) detect group differences in the ratings of emotional faces, as well as (2) determine more thoroughly the influence of anxiety symptoms, subjective feelings of fear, and specific caregiver-deprived and adoption-related variables (age at placement in foster care/ institution, age at adoption, length of time spent in foster care/institution) on medial temporal lobe function. A larger sample would help to investigate links between caregiver deprivation, emotional neglect, and ratings of emotional faces more in depth, and would be helpful for the finegrained analysis of potential moderating factors. Finally, the study was underpowered to examine differences be- 
tween boys and girls on behavioral and neural responses. This question will need to be addressed in the future, based on evidence that facial expression processing, as well as amygdala response to fearful faces, varies between healthy boys and girls (Killgore, Oki, \& Yurgelun-Todd, 2001; McClure, 2000). However, in the few studies of caregiverdeprived and maltreated youths, except for that of Tupler and De Bellis (2006), no differences were found between boys and girls in emotional processing (Pollak et al., 2000; Pollak \& Sinha, 2002; Vorria et al., 2006; Wismer-Fries \& Pollak, 2004) or medial temporal lobe alterations (Mehta et al., 2009). Unfortunately, the sample size in these studies was also small. Thus, studying a larger sample would help measure more reliably the influence of gender on emotional processing and underlying neural systems in caregiver-deprived and emotionally neglected youths.

A second important limitation concerns the heterogeneity in the caregiver deprivation history of participants in the experimental group. Some of the caregiver-deprived youths were adopted from U.S. foster care, some from international orphanages. Hence, some were raised in a U.S. environment, whereas others were raised in a foreign environment. Moreover, although poor conditions of care in orphanages of all these areas were reported (Human Rights Watch, 1998; K. Lee, 2000; Lien, Meyer, \& Winick, 1977; Mason \& Narad, 2005; Winick, Meyer, \& Harris, 1975), it remains that postinstitutionalized youths were adopted from four different countries: China, Korea, Russia, and Siberia. All of these factors may have yielded different experiences of deprivation, which may have influenced the medial temporal lobe findings. However, losing a primary caregiver as well as being separated from one's original family and placed in either foster care or institutional care are all stressful experiences. All types of stressors, whether psychological (e.g., neglect, caregiver deprivation) or physical (e.g., fasting, extreme cold), trigger the same determinants of the stress response, such as stress-related hormones (e.g., cortisol) and neurotransmitters (e.g., serotonin), which influence the brain. Medial temporal lobe structure and function are particularly sensitive to the influence of these stress determinants (Lupien, Maheu, Tu, Fiocco, \& Schramek, 2007; Meaney, 2001; Teicher et al., 2002). Since all youths from the experimental group experienced similar stressful situations (caregiver deprivation, emotional neglect) and none of them received treatment, we expected to observe similar medial temporal lobe abnormalities in all of these participants. Nevertheless, future studies should investigate the influence of types of institutional placement (family foster care vs. orphanages), types of placement environment (same culture as adoptive home vs. foreign culture), and countries from which youths are adopted in order to determine more thoroughly whether or not these variables have specific modulatory effects on brain function.

\section{AUTHOR NOTE}

The present study was supported by a National Alliance for Research on Schizophrenia and Depression Young Investigator Award and a postdoctoral fellowship from the Fonds de la Recherche en Santé du Québec to F.S.M., by funding from Edna Bennett Pierce, by NIMH Grants 52135 and 74374 to M.D., and by the NIMH Intramural Research Program. The authors thank the anonymous reviewers for their helpful comments in revising the manuscript. Address correspondence to F. S. Maheu, Research Centre, CHU Ste-Justine and Psychiatry Department, Faculty of Medicine, University of Montreal, 3175 Côte-Ste-Catherine, Montreal, QC, H3T 1C5 Canada (e-mail: francoise.maheu@umontreal.ca).

\section{REFERENCES}

AdOLPHS, R. (2003). Cognitive neuroscience of human social behaviour. Nature Reviews Neuroscience, 4, 165-178.

American Academy of Pediatrics (2000). Developmental issues for young children in foster care. Pediatrics, 106, 1145-1150.

Armanini, M. P., Hutchins, C., Stein, B. A., \& Sapolsky, R. M. (1990). Glucocorticoid endangerment of hippocampal neurons is NMDA-receptor dependent. Brain Research, 532, 7-12.

Armony, J. L., Corbo, V., Clément, M. H., \& Brunet, A. (2005). Amygdala response in patients with acute PTSD to masked and unmasked emotional facial expressions. American Journal of Psychiatry, 162, 1961-1963.

BaAs, D., Aleman, A., \& KAHn, R. S. (2004). Lateralization of amygdala activation: A systematic review of functional neuroimaging studies. Brain Research Reviews, 45, 96-103.

Bannerman, D., Rawlins, J., McHugh, S., Deacon, R., Yee, B., BAST, T., ET AL. (2004). Regional dissociations within the hippocampus -Memory and anxiety. Neuroscience \& Biobehavioral Reviews, 28, 273-283.

Birmaher, B., Khetarpal, S., Brent, D., Cully, M., Balach, L., Kaufman, J., \& Neer, S. M. (1997). The Screen for Child Anxiety Related Emotional Disorders (SCARED): Scale construction and psychometric characteristics. Journal of the American Academy of Child \& Adolescent Psychiatry, 36, 545-553.

Bremner, J. D. (2007). Functional neuroimaging in post-traumatic stress disorder. Expert Reviews of Neurotherapeutics, 7, 393-405.

Bremner, J. D., Narayan, M., Staib, L. H., Southwick, S. M., McGlashan, T., \& Charney, D. S. (1999). Neural correlates of memories of childhood sexual abuse in women with and without posttraumatic stress disorder. American Journal of Psychiatry, 156, 1787-1795.

Bremner, J. D., Vermetten, E., Schmahl, C., Vaccarino, V., Vythilingam, M., AfZal, N., ET Al. (2005). Positron emission tomographic imaging of neural correlates of a fear acquisition and extinction paradigm in women with childhood sexual-abuse-related post-traumatic stress disorder. Psychological Medicine, 35, 791-806.

Bremner, J. D., Vythilingam, M., Vermetten, E., Southwick, S. M., McGlashan, T., Nazeer, A., ET Al. (2003). MRI and PET study of deficits in hippocampal structure and function in women with childhood sexual abuse and posttraumatic stress disorder. American Journal of Psychiatry, 160, 924-932.

Bremner, J. D., Vythilingam, M., Vermetten, E., Southwick, S. M., McGlashan, T., Staib, L. H., ET AL. (2003). Neural correlates of declarative memory for emotionally valenced words in women with posttraumatic stress disorder related to early childhood sexual abuse. Biological Psychiatry, 53, 879-889.

Carrion, V. G., Weems, C. F., \& Reiss, A. L. (2007). Stress predicts brain changes in children: A pilot longitudinal study on youth stress, posttraumatic stress disorder, and the hippocampus. Pediatrics, 119, 509-516.

Chugani, H. T., Behen, M. E., Muzik, O., Juhasz, C., Nagy, F., \& Chugani, D. C. (2001). Local brain functional activity following early deprivation: A study of postinstitutionalized Romanian orphans. NeuroImage, 14, 1290-1301.

Cicchetтi, D., \& Toth, S. L. (2005). Child maltreatment. Annual Reviews in Clinical Psychology, 1, 409-438.

DAvidson, R. J. (2004). Well-being and affective style: Neural substrates and biobehavioural correlates. Philosophical Transactions of the Royal Society B, 359, 1395-1411.

De Bellis, M. D. (2005). The psychobiology of neglect. Child Maltreatment, 10, 150-172.

De Bellis, M. D., Keshavan, M. S., Shifflett, H., Iyengar, S., Beers, S. R., Hall, J., ET AL. (2002). Brain structures in pediatric maltreatment-related posttraumatic stress disorder: A sociodemographically matched study. Biological Psychiatry, 52, 1066-1078. 
De Bellis, M. D., \& Thomas, L. A. (2003). Biologic findings of posttraumatic stress disorder and child maltreatment. Current Psychiatry Reports, 5, 108-117.

Denson, T. F., Pedersen, W. C., Ronquillo, J., \& Nandy, A. S. (2008). The angry brain: Neural correlates of anger, angry rumination, and aggressive personality. Journal of Cognitive Neuroscience, 21, 734-744.

Dolan, M. C., \& Fullam, R. S. (2009). Psychopathy and functional magnetic resonance imaging blood oxygenation level-dependent responses to emotional faces in violent patients with schizophrenia. Biological Psychiatry, 66, 570-577. doi:10.1016/j.biopsych.2009.03.019

DolCos, F., LABAR, K., \& CABEZA, R. (2004). Interaction between the amygdala and the medial temporal lobe memory system predicts better memory for emotional events. Neuron, 42, 855-863.

Dozier, M., Albus, K., Fisher, P. A., \& Sepulveda, S. (2002). Interventions for foster parents: Implications for developmental theory. Development \& Psychopathology, 14, 843-860.

Dozier, M., Manni, M., Gordon, M. K., Peloso, E., Gunnar, M. R., Stovall-McClough, K. C., ET AL. (2006). Foster children's diurnal production of cortisol: An exploratory study. Child Maltreatment, 11, 189-197.

Duke, P. M., LitT, I. F., \& Gross, R. T. (1980). Adolescents' selfassessment of sexual maturation. Pediatrics, 66, 918-920.

Ekman, P., \& Friesen, W. V. (1976). Pictures of facial affect. Palo Alto, CA: Consulting Psychologists Press.

Eluvathingal, T. J., Chugani, H. T., Behen, M. E., Juhász, C., MuZIK, O., MAQBOoL, M., ET AL. (2006). Abnormal brain connectivity in children after early severe socioemotional deprivation: A diffusion tensor imaging study. Pediatrics, 117, 2093-2100.

Ernst, M., Maheu, F. S., Schroth, E., Hardin, J., Green-Golan, L., CAmeron, J., ET AL. (2007). Amygdala function in adolescents with congenital adrenal hyperplasia: A model for the study of early steroid abnormalities. Neuropsychologia, 45, 2104-2113.

Friston, K., Mechelli, A., Turner, R., \& Price, C. (2000). Nonlinear responses in fMRI: The balloon model, Volterra kernels, and other hemodynamics. NeuroImage, 12, 466-477.

Fusar-Poli, P., Placentino, A., Carletti, F., Allen, P., Landi, P., AbBamonte, M., ET AL. (2009). Laterality effect on emotional faces processing: ALE meta-analysis of evidence. Neuroscience Letters, 452, 262-267.

Greenhouse, S. W., \& Geisser, S. (1959). On methods in the analysis of profile data. Psychometrika, 24, 95-112.

Gunnar, M. R., Bruce, J., \& Grotevant, H. D. (2000). International adoption of institutionally reared children: Research and policy. Development \& Psychopathology, 12, 677-693.

Guyer, A. E., Monk, C. S., McClure-Tone, E. B., Nelsom, E. E., Roberson-NaY., R., AdLer, A. D., ET AL. (2008). A developmental examination of amygdala response to facial expressions. Journal of Cognitive Neuroscience, 20, 1565-1582.

Halfon, N., Mendonca, A., \& Berkowitz, G. (1995). Health status of children in foster care: The experience of the Center for the Vulnerable Child. Archives of Pediatrics \& Adolescent Medicine, 149, 386-392.

Hariri, A. R., Bookheimer, S. Y., \& Mazziotta, J. C. (2000). Modulating emotional responses: Effects of a neocortical network on the limbic system. NeuroReport, 11, 43-48.

Hollingshead, A. B. (1973). Four-factor index of social status. New Haven, CT: Yale University Press.

Holt, D. J., Weiss, A. P., Rauch, S. L., Wright, C. I., Zalesak, M., GoFf, D. C., ET AL. (2005). Sustained activation of the hippocampus in response to fearful faces in schizophrenia. Biological Psychiatry, 57, 1011-1019.

Human Rights Watch (1998). Abandoned to the state: Cruelty and neglect in Russian orphanages. New York: Author.

Kaufman, J., Birmaher, B., Brent, D., Rao, U., Flynn, C., Moreci, P., ET AL. (1997). Schedule for affective disorders and schizophrenia for school-age children - present and lifetime version (K-SADS-PL): Initial reliability and validity data. Journal of the American Academy of Child \& Adolescent Psychiatry, 36, 980-988.

Kaufman, J., Plotsky, P. M., Nemeroff, C. B., \& Charney, D. S. (2000). Effects of early adverse experiences on brain structure and function: Clinical implications. Biological Psychiatry, 48, 778-790.

Killgore, W. D., OKI, M., \& Yurgelun-TodD, D. A. (2001). Sex- specific developmental changes in amygdala responses to affective faces. NeuroReport, 12, 427-433.

Kim, S.-E., Kim, J.-W., Kim, J.-J., Jeong, B. S., Choi, E. A., Jeong, Y.-G., ET AL. (2007). The neural mechanism of imagining facial affective expression. Brain Research, 1145, 128-137.

Kovacs, M. (1985). The Children's Depression Inventory (CDI). Psychopharmacology Bulletin, 21, 995-998.

Kreppner, J. M., Rutter, M., Beckett, C., Castle, J., Colvert, E., Groothues, C., ET AL. (2007). Normality and impairment following profound early institutional deprivation: A longitudinal follow-up into early adolescence. Developmental Psychology, 43, 931-946.

LeDoux, J. E. (2000). Emotion circuits in the brain. Annual Reviews of Neuroscience, 23, 155-184.

Lee, B. T., Seok, J. H., Lee, B. C., Cho, S. W., Yoon, B. J., Lee, K. U., ET AL. (2008). Neural correlates of affective processing in response to sad and angry facial stimuli in patients with major depressive disorder. Progress in Neuropsychopharmacology \& Biological Psychiatry, 32, 778-785.

LEE, K. (2000). Crying patterns of Korean infants in institutions. Child Care \& Health Development, 26, 217-228.

Lien, N. M., MeYer, K. K., \& WinicK, M. (1977). Early malnutrition and late adoption: A study of their effects on the development of Korean orphans adopted into American families. American Journal of Clinical Nutrition, 30, 1734-1739.

Lupien, S. J., Maheu, F. S., Tu, M., Fiocco, A., \& Schramek, T. E. (2007). The effects of stress and stress hormones on human cognition: Implications for the field of brain and cognition. Brain \& Cognition, 65, 209-237.

Maheu, F. S., Mazzone, L., Merke, D. P., Keil, M. F., Stratakis, C. A., Pine, D. S., \& ERnst, M. (2008). Altered amygdala and hippocampus function in adolescents with hypercortisolemia: An fMRI study of Cushing syndrome. Development \& Psychopathology, 20, 1177-1189.

Mason, P., \& NARAD, C. (2005). International adoption: A health and developmental prospective. Seminars in Speech \& Language, 26, 1-9.

Masten, C. L., Guyer, A. E., Hodgdon, H. B., McClure, E. B., CharNEY, D. S., ERNST, M., ET AL. (2008). Recognition of facial emotions among maltreated children with high rates of post-traumatic stress disorder. Child Abuse \& Neglect, 32, 139-153.

MCClure, E. B. (2000). A meta-analytic review of sex differences in facial expression processing and their development in infants, children, and adolescents. Psychological Bulletin, 126, 424-453.

McClure, E. B., Monk, C. S., Nelson, E. E., PArrish, J. M., Adler, A., BLAIR, R. J. R., ET AL. (2007). Abnormal attention modulation of fear circuit function in pediatric generalized anxiety disorder. Archives of General Psychiatry, 64, 97-106.

McClure, E. B., Monk, C. S., Nelson, E. E., Zarahn, E., LeibenLUFT, E., BILDER, R. M., ET AL. (2004). A developmental examination of gender differences in brain engagement during evaluation of threat. Biological Psychiatry, 55, 1047-1055.

McGowan, P. O., Sasaki, A., D'Alessio, A. C., Dymov, S., LaBONTÉ, B., SZYF, M., ET AL. (2009). Epigenetic regulation of the glucocorticoid receptor in human brain associates with childhood abuse. Nature Neuroscience, 12, 342-348.

Meaney, M. J. (2001). Maternal care, gene expression, and the transmission of individual differences in stress reactivity across generations. Annual Reviews of Neuroscience, 24, 1161-1192.

Mehta, M. A., Golembo, N. I., Nosarti, C., Colvert, E., Mota, A., Williams, S. C. R., ET AL. (2009). Amygdala, hippocampal and corpus callosum size following severe early institutional deprivation: The English and Romanian Adoptees Study pilot. Journal of Child Psychology \& Psychiatry, 50, 943-951. doi:10.1111/j.1469 $-7610.2009 .02084 . \mathrm{x}$

Monk, C. S., McClure, E. B., Nelson, E. E., Zarahn, E., Bilder, R. M., Leibenluft, E., Et AL. (2003). Adolescent immaturity in attention-related brain engagement to emotional facial expressions. NeuroImage, 20, 420-428.

Monk, C. S., Nelson, E. E., McClure, E. B., MogG, K., Bradley, B. P., LeIBENLUfT, E., ET AL. (2006). Ventrolateral prefrontal cortex activation and attentional bias in response to angry faces in adolescents with generalized anxiety disorder. American Journal of Psychiatry, 163, 1091-1097.

Monk, C. S., Telzer, E. H., Mogg, K., Bradley, B. P., Mai, X., 
Louro, H. M. C., ET AL. (2008). Amygdala and ventrolateral prefrontal cortex activation to masked angry faces in children and adolescents with generalized anxiety disorder. Archives of General Psychiatry, 65, 568-576.

Murphy, F. C., Nimmo-Smith, I., \& Lawrence, A. D. (2003). Functional neuroanatomy of emotions: A meta-analysis. Cognitive, Affective, \& Behavioral Neuroscience, 3, 207-233.

Nelson, E. E., McClure, E. B., Monk, C. S., Zarahn, E., LeibenLuft, E., Pine, D. S., \& Ernst, M. (2003). Developmental differences in neuronal engagement during implicit encoding of emotional faces: An event-related fMRI study. Journal of Child Psychology \& Psychiatry, 44, 1015-1024.

Nickman, S. L., Rosenfeld, A. A., Fine, P., Macintyre, J. C., Pilowsky, D. J., Howe, R. A., ET AL. (2005). Children in adoptive families: Overview and update. Journal of the American Academy of Children \& Adolescent Psychiatry, 44, 987-995.

Park, J. Y., Gu, B. M., Kang, D. H., Shin, Y. W., Choi, C. H., Lee, J. M., \& Kwon, J. S. (2008). Integration of cross-modal emotional information in the human brain: An fMRI study. Cortex, 46, 161-169. doi: 10.1016/j.cortex.2008.06.008

Pears, K. [C.], \& Fisher, P. A. (2005a). Developmental, cognitive, and neuropsychological functioning in preschool-aged foster children: Associations with prior maltreatment and placement history. Developmental \& Behavioral Pediatrics, 26, 112-122.

Pears, K. C., \& Fisher, P. A. (2005b). Emotion understanding and theory of mind among maltreated children in foster care: Evidence of deficits. Development \& Psychopathology, 17, 47-65.

Pérez-Edgar, K., Roberson-Nay, R., Hardin, M. G., Poeth, K., Guyer, A. E., Nelson, E. E., ET AL. (2007). Attention alters neural responses to evocative faces in behaviorally inhibited adolescents. NeuroImage, 35, 1538-1546.

Phan, K. L., Wager, T., Taylor, S. F., \& Liberzon, I. (2002). Functional neuroanatomy of emotion: A meta-analysis of emotion activation studies in PET and fMRI. NeuroImage, 16, 331-348.

PINE, D. S. (2003). Developmental psychobiology and response to threats: Relevance to trauma in children and adolescents. Biological Psychiatry, 53, 796-808.

PINE, D. S. (2007). Research review: A neuroscience framework for pediatric anxiety disorders. Journal of Child Psychology \& Psychiatry, 48, 631-648.

Pine, D. S., \& Cohen, J. A. (2002). Trauma in children and adolescents: Risk and treatment of psychiatric sequelae. Biological Psychiatry, 51, 519-531.

Pine, D. S., Cohen, P., Gurley, D., Brook, J., \& Ma, Y. (1998). The risk for early-adulthood anxiety and depressive disorders in adolescents with anxiety and depressive disorders. Archives of General Psychiatry, 55, 56-64.

Pine, D. S., Mogg, K., Bradley, B. P., Montgomery, L., Monk, C. S., McClure, E., ET AL. (2005). Attention bias to threat in maltreated children: Implications for vulnerability to stress-related psychopathology. American Journal of Psychiatry, 162, 291-296.

PollaK, S. D. (2003). Experience-dependent affective learning and risk for psychopathology in children. Annals of the New York Academy of Sciences, 1008, 102-111.

PolLAK, S. D. (2005). Early caregiver deprivation and mechanisms of plasticity: Integrating affective neuroscience with developmental approaches to psychopathology. Development \& Psychopathology, 17, 735-752.

Pollak, S. D., Cicchetti, D., Hornung, K., \& Reed, A. (2000). Recognizing emotion in faces: Developmental effects of child abuse and neglect. Developmental Psychology, 36, 679-688.

Pollak, S. D., \& SinHa, P. (2002). Effects of early experience on children's recognition of facial displays of emotion. Developmental Psychology, 38, 784-791.

Pollak, S. D., \& Tolley-Schell, S. A. (2003). Selective attention to facial emotion in physically abused children. Journal of Abnormal Psychology, 112, 323-338.

Reinders, A. A. T. S., Gläscher, J., De Jong, J. R., Willemsen, A. T. M., DEN Boer, J. A., \& BüCHEL, C. (2006). Detecting fear and neutral faces: BOLD latency differences in amygdala-hippocampal junction. NeuroImage, 33, 805-814.

Roberson-Nay, R., McClure, E. B., Monk, C. S., Nelson, E. E.,
Guyer, A. E., Froom, S., ET AL. (2006). Increased amygdala activity during successful memory encoding in adolescent major depressive disorder: An fMRI study. Biological Psychiatry, 60, 966-973.

Rutter, M., Colvert, E., Kreppner, J., Beckett, C., Castle, J., Groothues, C., ET AL. (2007). Early adolescent outcomes for institutionally-deprived and non-deprived adoptees. I: Disinhibited attachment. Journal of Child Psychology \& Psychiatry, 48, 17-30.

Rutter, M., \& THE English and Romanian Adoptees (ERA) Study TEAM (1998). Developmental catch-up, and deficit, following adoption after severe global early privation. Journal of Child Psychology \& Psychiatry, 39, 465-476.

Rutter, M., O'Connor, T. G., \& THE English and Romanian AdopTEES (ERA) StUdy TEAm (2004). Are there biological programming effects for psychological development? Findings from a study of Romanian adoptees. Developmental Psychology, 40, 81-94.

SANCHEZ, M. M. (2006). The impact of early adverse care on HPA axis development: Nonhuman primate models. Hormones \& Behavior, 50, 623-631.

Sanchez, M. M., Ladd, C. O., \& Plotsky, P. M. (2001). Early adverse experience as a developmental risk factor for later psychopathology: Evidence from rodent and primate models. Development \& Psychopathology, 13, 419-449.

Sawyer, M. G., Carbone, J. A., Searle, A. K., \& Robinson, P. (2007). The mental health and wellbeing of children and adolescents in homebased foster care. Medical Journal of Australia, 186, 181-184.

Smucker, M. R., Craighead, W. E., Craighead, L. W., \& Green, B. J. (1986). Normative and reliability data for the children's depression inventory. Journal of Abnormal Child Psychology, 14, 25-39.

Szeszko, P. R., Robinson, D., Alvir, J. M. J., Bilder, R. M., LenCz, T., AshtARI, M., ET AL. (1999). Orbital frontal and amygdala volume reductions in obsessive-compulsive disorder. Archives of General Psychiatry, 56, 913-919.

Szeszko, P. R., Strous, R. D., Goldman, R. S., Ashtari, M., Knuth, K. H., Lieberman, J. A., \& Bilder, R. M. (2002). Neuropsychological correlates of hippocampal volumes in patients experiencing a first episode of schizophrenia. American Journal of Psychiatry, 159, 217-226.

TANNER, J. M., \& Whitehouse, R. H. (1976). Clinical longitudinal standards for height, weight, height velocity, weight velocity, and stages of puberty. Archives of Disease in Childhood, 51, 170-179.

Teicher, M. H., Andersen, S. L., Polcari, A., Anderson, C. M., \& Navalta, C. P. (2002). Developmental neurobiology of childhood stress and trauma. Psychiatry Clinics of North America, 25, 397-426.

Tupler, L. A., \& De Bellis, M. D. (2006). Segmented hippocampal volume in children and adolescents with posttraumatic stress disorder. Biological Psychiatry, 59, 523-529.

Tyler, S., Allison, K., \& Winsler, A. (2006). Child neglect: Developmental consequences, intervention, and policy implications. Child \& Youth Care Forum, 35, 1-20.

Vorria, P., Papaligoura, Z., Sarafidou, J., Kopakaki, M., Dunn, J., VAN IJZENDOORN, M. H., ET AL. (2006). The development of adopted children after institutional care: A follow-up study. Journal of Child Psychology \& Psychiatry, 47, 1246-1253.

WeChSLER, D. (1999). Wechsler Abbreviated Scale of Intelligence. San Antonio, TX: Psychological Corporation.

WinicK, M., MeYER, K. K., \& HARRIs, R. C. (1975). Malnutrition and environmental enrichment by early adoption. Science, 190, 1173-1175.

Winston, J., Strange, B., O'Doherty, J., \& Dolan, R. (2002). Automatic and intentional brain responses during evaluation of trustworthiness of faces. Nature Neuroscience, 5, 277-283.

Wismer-Fries, A. B., \& PollaK, S. D. (2004). Emotion understanding in postinstitutionalized Eastern European children. Development \& Psychopathology, 16, 355-369.

Zarahn, E., Aguirre, G. K., \& D'Esposito, M. (1997). Empirical analyses of BOLD fMRI statistics: I. Spatially unsmoothed data collected under null-hypothesis conditions. NeuroImage, 5, 179-197.

ZHOU, M., \& BAUDRY, M. (2006). Developmental changes in NMDA neurotoxicity reflect developmental changes in subunit composition of NMDA receptors. Journal of Neuroscience, 26, 2956-2963.

(Manuscript received February 26, 2009; revision accepted for publication July 27, 2009.) 